\title{
THE INFLUENCE OF TEXTURE ON THE SHAPE- MEMORY EFFECT IN POLYCRYSTALS
}

\author{
Y. C. SHU and K. BHATTACHARYA $\dagger$ \\ Division of Engineering and Applied Science, 104-44, California Institute of Technology, Pasadena, \\ CA 91125 , U.S.A.
}

(Received 29 October 1997; accepted 18 May 1998)

\begin{abstract}
A model is developed to show that texture is a crucial factor in determining the shape-memory effect in polycrystals. In particular, it is established that texture is the reason why the strains recoverable in $\mathrm{Ti}-\mathrm{Ni}$ are so much larger than those in $\mathrm{Cu}$-based shape-memory alloys in rolled, extruded and drawn specimens. Further, it is shown that both these materials recover relatively small strains in sputter-deposited thin films due to unfavorable texture. It is found that even the qualitative behavior of combined tensiontorsion can critically depend on the texture. The results are in good agreement with experimental observations. Finally, textures are suggested for improved shape-memory effect. (C) 1998 Acta Metallurgica Inc. Published by Elsevier Science Ltd. All rights reserved.
\end{abstract}

\section{INTRODUCTION}

Shape-memory effect (SME) is a phenomenon where deformation suffered below a critical temperature can be recovered on heating. About 20-30 alloys are known to exhibit SME in single crystals. However, the degree to which they retain their shape-memory behavior in polycrystals is widely varied. Some materials have good shape-memory behavior as single crystals but little or none as polycrystals, while others display good SME even as polycrystals. Bhattacharya and Kohn [1] have argued that crystallographic symmetry is a very important factor in determining the recoverable strains in polycrystals: alloys which undergo small change in symmetry (cubic to tetragonal or trigonal) have virtually no recoverable strains in polycrystals, while alloys which undergo large change in symmetry (cubic to orthorhombic or monoclinic) always recover at least some strain even in polycrystals. While this explains much experimental observation, it fails to make any distinction within the latter group of alloys. In particular, it is unable to explain why $\mathrm{Ti}-\mathrm{Ni}$ is so much better than $\mathrm{Cu}$-based SMAs (Table 1) since both undergo cubic-monoclinic transformation and have similar transformation strains. They speculated on, but did not systematically explore, the role of texture.

Typically, shape-memory materials are prepared by casting followed by hot-working (rolling for strips or drawing for wires) followed by heat treatment. Therefore, it is natural to expect that the

†To whom all correspondence should be addressed. polycrystals are textured and this has been experimentally confirmed in $\mathrm{Ti}-\mathrm{Ni}$ rolled sheets [8,1621]; $\mathrm{Ti}-\mathrm{Ni}$ rods and $\mathrm{Ti}-\mathrm{Ni}-\mathrm{Cu}$ wires $[22,23] ; \mathrm{Cu}-$ $\mathrm{Zn}-\mathrm{Al}$ rolled sheets [24-26]; and $\mathrm{Cu}-\mathrm{Zn}-\mathrm{Al}$ drawn wires [27]. Other processing means like rapid solidification [8,28-30] and sputtering [31] also endow the polycrystal with texture.

In this paper, we explore the effect of texture on SME by extending the framework of Bhattacharya and Kohn [32]. We use "recoverable strain" as a measure of SME and develop a model to estimate it for a polycrystal with given texture. A geometric point of view is adopted for brevity. We note that it is possible to justify this geometric picture using energy minimization, and we refer interested readers to Refs $[32,33]$ for details. We also note that the strains recoverable by superelasticity or pseudoelasticity are essentially identical to those recoverable by SME; so our model also provides a means to evaluate the effect of texture on superelasticity. Using this model, we show that texture is a very important reason for the difference between $\mathrm{Ti}-\mathrm{Ni}$ and $\mathrm{Cu}$-based SMAs: the texture that develops during rolling or drawing is very desirable from the point of view of SME in $\mathrm{Ti}-\mathrm{Ni}$ while these textures are undesirable in $\mathrm{Cu}$-based alloys. We also find that sputtering textures in both $\mathrm{Ti}-\mathrm{Ni}$ and $\mathrm{Cu}-$ based shape-memory thin films are not favorable for large recoverable strain. This is consistent with experimental observations. Finally, we explore in some detail the predicted recoverable strains for various textures in an attempt to recommend textures for large SME. 
Table 1. Experimental observations of the recoverable strains in some $\mathrm{Cu}$-based SMAs and $\mathrm{Ti}-\mathrm{Ni}$

\begin{tabular}{|c|c|c|}
\hline \multirow[t]{2}{*}{ SMA } & \multicolumn{2}{|r|}{ Recoverable strain } \\
\hline & Single crystals & Polycrystals \\
\hline $\begin{array}{l}\mathrm{Cu}-\mathrm{Zn}-\mathrm{Al} \\
\mathrm{Cu}-\mathrm{Al}-\mathrm{Ni}\end{array}$ & $2-9 \%$ depending on orientation $[2,3]$ & around $2 \%$ in general [4-7], but can be up to $6 \%$ in textured ribbons $[8,9]$ \\
\hline $\mathrm{Ti}-\mathrm{Ni}$ & $3-10 \%$ depending on orientation $[10,11]$ & $5-8 \%$ in drawn wires and rolled sheets $[12-15]$ \\
\hline
\end{tabular}

The source of the SME is a martensitic phase transformation. This gives rise to microstructure of martensitic variants below the transformation temperature, and certain deformations are recoverable because they are accomplished not by slip or the motion of dislocations but by rearrangement of the microstructure. In a single crystal, it is relatively easy to calculate the recoverable strains and we characterize these using $\mathscr{S}$ - the set of recoverable strains in a single crystal-in Section 2.1. In a polycrystal, each grain is constrained by its neighbors as it tries to deform by rearranging its microstructure. Therefore, the set $\mathscr{P}$ - the set of recoverable strains in a polycrystal-described in Section 2.2 is rather difficult to calculate. Further, its calculation requires knowledge of the shape and orientation of each grain which is difficult to obtain. Finally, even if we measure the exact texture and solve the problem for a specific specimen, the resulting set is valid only for that specimen; we have to repeat the process for each specimen. Therefore, we need an easily computable, but reasonable estimate of this set which can use readily measurable and characterizable information about the texture. This is accomplished using bounds - the inner or the Taylor bound $\mathscr{P}_{\mathrm{i}}$ and the outer or the Sachs bound $\mathscr{P}_{\mathrm{o}}$-in Section 2.3. The inner bound is obtained by imposing the same strain on each grain while the outer bound is obtained by ignoring compatibility between grains.

We specialize on specific loads in Section 3. Uniaxial tension or compression is considered as an example of homogeneous loads and combined tension-torsion as an example of inhomogeneous loads. We show that under uniaxial loading of single crystals, our model essentially coincides with that of Saburi and Nenno [34]. In polycrystals, we again provide an inner bound and an outer bound of the recoverable strain. For monoclinic martensites, we also provide an inner estimate.

We believe that the inner bound is in fact a reasonable estimate of the actual recoverable strains. An example is provided to justify this in Section 3.2. Bhattacharya and Kohn [32] as well as Kohn and Niethammer [35] have derived rigorous results in dimensionally reduced problems to support this argument. Therefore, we use the inner bound, which can be rewritten as a linear programming problem, as our fundamental tool for evaluating the effect of texture.
The outer bound, on the other hand, is often a large overestimate. In uniaxial tension, the outer bound is the average of the strain of the most favorable martensitic variant in each grain. Various authors [17,19] have used this as a model for the recoverable strain and have predicted almost twice the observed recoverable strain.

We extend these ideas to the behavior of very thin films in Section 4. Shape-memory materials have the largest energy output per unit volume per cycle of known actuator systems [36]. This fact, together with the enhanced rate of heat transfer in thin films, makes these alloys ideal for microactuator, micropump and for microelectromechanical systems (MEMS) applications and this has motivated many experimental efforts (see, e.g. Refs [3740]). All of these efforts have concentrated on $\mathrm{Ti}-$ Ni-based alloys and use sputtering to produce polycrystalline films. However, it is not clear that $\mathrm{Ti}-\mathrm{Ni}$ is the ideal material in thin films. It is well known that microstructure can be significantly different in thin films as compared to bulk materials. Recently Bhattacharya and James [41] have developed a theory of martensitic thin films which captures this effect. We discuss the extension of this theory to polycrystalline thin films in this section.

We use our models to explore the effect of texture on recoverable strains in uniaxial tension in Section 5 and in combined tension-torsion in Section 6. Recently Zhao and Beyer $[20,21]$ as well as other groups [17-19] have measured both the recoverable strain and the texture of rolled $\mathrm{Ti}-\mathrm{Ni}$ and closely related materials. We calculate the recoverable strains for their observed texture and compare them with experimental observations in Section 5.1. Both $\mathrm{Ti}-\mathrm{Ni}$ and $\mathrm{Cu}$-based alloys, and, in fact, most SMAs, have a body-centeredcubic (b.c.c.) superlattice structure in their parent phases. We calculate recoverable strains for typical b.c.c. rolling and drawing textures, and also for textures formed by rapid solidification and sputtering. Figures 9 and 10 highlight the striking contrast in SME between $\mathrm{Ti}-\mathrm{Ni}$ and $\mathrm{Cu}$-based alloys in rolling textures. The results for various other textures are shown in Table 5. In Section 6, we find that even the qualitative behavior of combined tension-torsion can depend on the texture as shown in Fig. 11. We conclude in Section 7 with a discussion. 
Table 2. Transformation strains associated with different changes of symmetry. Only $e^{(1)}$ is shown here; the rest $e^{(2)}, \ldots, e^{(k)}$ can be obtained from $e^{(1)}$ by symmetry by permuting the basis. In Monoclinic-I, the axis of monoclinic symmetry corresponds to $\langle 110\rangle_{\text {cubic }}$ while in Monoclinic-II, the axis of monoclinic symmetry corresponds to $\langle 100\rangle_{\text {. }}$. We choose variant 1 so that $\delta>0$ (orthorhombic and monoclinic cases) and $\varepsilon>0$ (monoclinic case). The compositions of all alloys are given in atomic percentages unless otherwise specified

\begin{tabular}{|c|c|c|c|c|}
\hline Symmetry of martensite & $k$ & $\begin{array}{l}\text { Transformation } \\
\quad \text { strain } e^{(1)}\end{array}$ & Examples & Lattice parameters \\
\hline Tetragonal & 3 & $\left(\begin{array}{lll}\alpha & 0 & 0 \\
0 & \alpha & 0 \\
0 & 0 & \beta\end{array}\right)$ & $\mathrm{Ni}-36.8 \mathrm{Al}[42,43]$ & $\alpha=-0.0608, \beta=0.1302$ \\
\hline Trigonal & 4 & $\left(\begin{array}{lll}\beta & \alpha & \alpha \\
\alpha & \beta & \alpha \\
\alpha & \alpha & \beta\end{array}\right)$ & Ti-50.5Ni (R-phase) [44] & $\alpha=0.0047, \beta=0$ \\
\hline Orthorhombic & 6 & $\left(\begin{array}{lll}\alpha & \delta & 0 \\
\delta & \alpha & 0 \\
0 & 0 & \beta\end{array}\right)$ & $\begin{array}{l}\gamma_{1}^{\prime} \mathrm{Cu}-14 \mathrm{Al}-4 \mathrm{Ni}(\mathrm{wt} \%)[45] \\
\quad \mathrm{Ti}-45 \mathrm{Ni}-5 \mathrm{Cu}[46]\end{array}$ & $\begin{array}{l}\alpha=0.0425, \beta=-0.0822, \delta=0.0194 \\
\alpha=0.0240, \beta=-0.0420, \delta=0.0310\end{array}$ \\
\hline Monoclinic-I & 12 & $\left(\begin{array}{lll}\alpha & \delta & \varepsilon \\
\delta & \alpha & \varepsilon \\
\varepsilon & \varepsilon & \beta\end{array}\right)$ & $\begin{array}{l}\mathrm{Ti}-49.8 \mathrm{Ni}[47,48] \\
\mathrm{Ti}-45 \mathrm{Ni}-5 \mathrm{Cu}[46]\end{array}$ & $\begin{array}{l}\alpha=0.0243, \beta=-0.0437, \delta=0.0580, \varepsilon=0.0427 \\
\alpha=0.0232, \beta=-0.0410, \delta=0.0532, \varepsilon=0.0395\end{array}$ \\
\hline Monoclinic-II & 12 & $\left(\begin{array}{ccc}\alpha+\varepsilon & \delta & 0 \\
\delta & \alpha-\varepsilon & 0 \\
0 & 0 & \beta\end{array}\right)$ & $\begin{array}{c}\mathrm{Cu}-15 \mathrm{Zn}-17 \mathrm{Al}[49] \\
\beta_{1}^{\prime} \mathrm{Cu}-14 \mathrm{Al}-4 \mathrm{Ni}(\mathrm{wt} \%)[50]\end{array}$ & $\begin{array}{c}\alpha=0.0483, \beta=-0.0907, \delta=0.0249, \varepsilon=0.0383 \\
\alpha=0.0442, \beta=-0.0822, \delta=0.0160, \varepsilon=0.06\end{array}$ \\
\hline
\end{tabular}

\section{RECOVERABLE STRAIN}

\subsection{Recoverable strain in a single crystal}

Consider a single crystal of austenite and choose this as the reference configuration, so the austenite has stress-free strain $e^{(0)}=0$. As it is cooled, it transforms to martensite. In SMAs, the austenite lattice has cubic symmetry while the martensite lattice has smaller symmetry such as tetragonal, trigonal, orthorhombic or monoclinic symmetry. This gives rise to $k$ symmetry-related variants of martensite. The transformation from the austenite to the $i$ th variant of martensite is described by the transformation strain $e^{(i)}$. It can be determined from the change of symmetry and lattice parameters as shown in Table 2. Schematically, we mark each transformation strain as a point $\bullet$ in Fig. 1 .
When a single crystal is below the transformation temperature, it can form microstructures by coherently mixing the variants of martensite. It deforms if it can by rearranging the microstructure. When it is subsequently heated, each variant reverts to the austenite and all the strain is recovered. Thus, the strains that a single crystal can recover are exactly those that are associated with a microstructure of martensitic variants. Thus, $\mathscr{S}$, the set of recoverable strains in a single crystal, is exactly the set of strains one can make by coherent microstructures of martensite.

Consider two variants of martensite - say 1 and 2 - and suppose that these variants are compatible or twin-related. In other words, assume that the transformation strains $e^{(1)}$ and $e^{(2)}$ satisfy the compatibility condition

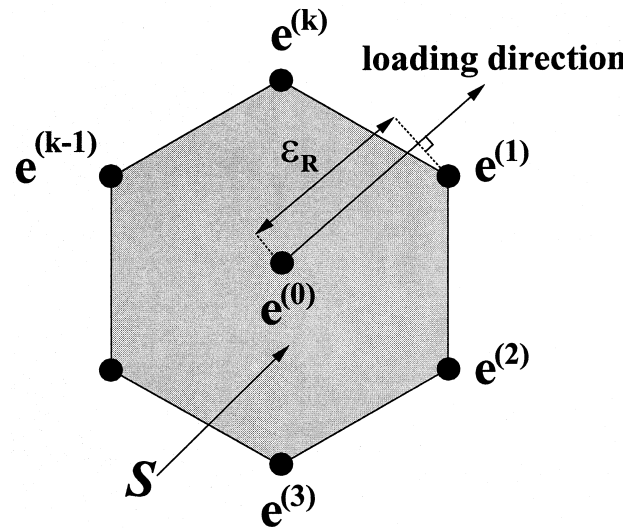

(a)

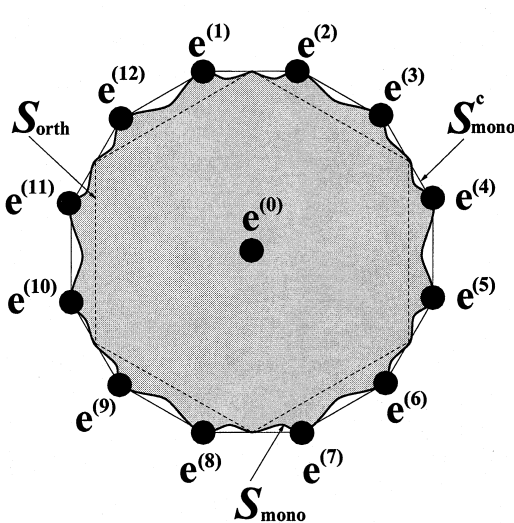

(b)

Fig. 1. (a) Recoverable strains in a single crystal when all pairs of martensitic variants are compatible. (b) The set of recoverable strains $\mathscr{S}_{\text {mono }}$ in a single crystal undergoing cubic-monoclinic transformation. This nonconvex set $\mathscr{S}_{\text {mono }}$ is bounded by orthorhombic $\mathscr{S}_{\text {orth }}$ and monoclinic $\mathscr{S}_{\text {mono }}^{\mathrm{c}}$ estimates. 


$$
e^{(1)}-e^{(2)}=\frac{1}{2}(a \otimes n+n \otimes a)
$$

for some vectors $a$ and $n$. Here $a \otimes n$ denotes the matrix with components $(a \otimes n)_{i j}=a_{i} n_{j}$. Then, we can form fine twins whose overall strain is the average of the transformation strains of these two variants. Geometrically, this average strain is a point on the line connecting these two variants, and by choosing the appropriate volume fraction we conclude that all strains on the line joining $e^{(1)}$ and $e^{(2)}$ are recoverable. Furthermore, if all the variants are pair-wise compatible, $\mathscr{S}$ is the convex hull of the transformation strains [51]; i.e.

$$
\mathscr{S}=\left\{e: e=\sum_{i=1}^{k} \mu_{i} e^{(i)}, \mu_{i} \geq 0, \sum_{i=1}^{k} \mu_{i}=1\right\}
$$

This is shown schematically as the shaded set in Fig. 1(a). The variants are pair-wise compatible in cubic-tetragonal, cubic-trigonal and cubic-orthorhombic transformation and we can obtain $\mathscr{S}$ in these cases.

The transformation strains of monoclinic martensites are not pair-wise compatible and the set of recoverable strains $\mathscr{S}_{\text {mono }}$ is not the convex hull of the transformation strains. This is because there are some strains that cannot be achieved by coherent microstructures of the different martensitic variants. Unfortunately, we do not know the precise set $\mathscr{S}_{\text {mono }}$, so we bound it using "orthorhombic" and "monoclinic" estimates as shown in Fig. 1(b). Let $\mathscr{S}_{\text {mono }}^{\mathrm{c}}$ be the convex hull of the monoclinic variants and let $\mathscr{S}_{\text {orth }}$ be the convex hull of a fictitious cubic-orthorhombic transformation obtained by setting $\varepsilon=0$ in $e^{(1)}, \ldots, e^{(12)}$. We can show that $\mathscr{S}_{\text {orth }} \subset \mathscr{S}_{\text {mono }} \subset \mathscr{S}_{\text {mono }}^{\mathrm{c}}[1,32]$.

Finally, shape-memory alloys are self-accommodating: they can undergo the austenite-martensite transformation with no apparent shape change. In other words, there is a microstructure of martensitic variants whose average strain is $e^{(0)}$ or $e^{(0)} \in \mathscr{S}$ [52] as also shown in Fig. 1.

Putting all this together, the situation is as follows. At high temperatures, the single crystal is in the austenite state with overall strain $e^{(0)}$. On cooling it transforms to self-accommodated martensite, also with strain $e^{(0)}$. We can now deform it freely as long as the strain is in the set $\mathscr{S}$. On subsequent heating, no matter where it is in the set $\mathscr{S}$ it reverts once again to austenite with overall strain $e^{(0)}$ recovering all imposed strain. If we deform beyond the set $\mathscr{S}$ at low temperature, elastic stresses build up resisting the deformation, resulting in dislocation motion or fracture and non-recoverability.

\subsection{Recoverable strain in a polycrystal}

We now turn to a polycrystal. A polycrystal is an aggregate of a great number of single crystal grains with different orientations. We describe the texture of a polycrystal by a rotation-valued function $R(x)$. $R(x)$ gives the orientation of the crystal at the point $x$ relative to some fixed reference crystal.

Suppose we start in the austenite and cool. Each grain transforms to a self-accommodated state. Suppose we now impose an overall strain $e_{\mathrm{o}}$, and ask if it is recoverable. Once again, it is if it can be accommodated by a microstructure of martensite in each grain. However, each grain has its own set of

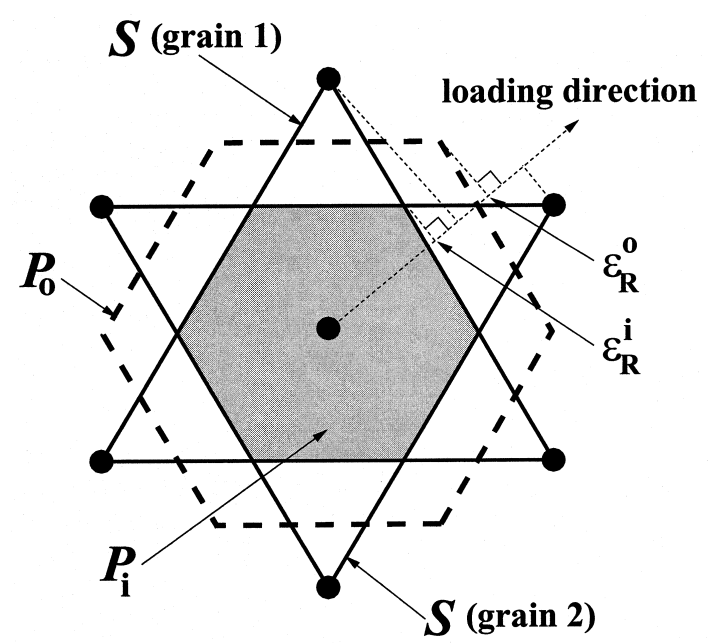

(a)

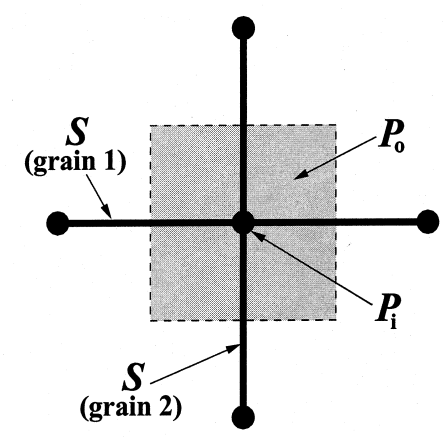

(b)

Fig. 2. (a) Recoverable strains in a polycrystal (made of two grains of equal volume fraction). Each grain has its own set of recoverable strains (the two triangles) obtained by a rotation of the basic set $\mathscr{S}$. The polycrystal can recover all strains inside the inner bound $\mathscr{P}_{\text {i }}$, but none outside the outer bound $\mathscr{P}_{\mathrm{o}}$. (b) Inner and outer bounds of recoverable strain can be wide apart when $\mathscr{S}$ has small dimension (schematically a line). 
recoverable strains

$$
\mathscr{S}(x)=R(x) \mathscr{S} R^{\mathrm{T}}(x)
$$

which is obtained by the rotation of the basic set $\mathscr{S}$ through $R(x)$ as shown in Fig. 2(a). Thus, in order to accommodate $e_{\mathrm{o}}$, the polycrystal has to find (a possibly inhomogeneous but compatible) strain field $e(x)$ that satisfies different constraints in different grains $(e(x) \in \mathscr{S}(x))$ and whose average is $e_{\mathrm{o}}$. Thus, $\mathscr{P}$, the set of recoverable strains in a polycrystal, is given by

$$
\begin{array}{r}
\mathscr{P}=\left\{e_{\mathrm{o}}: e_{\mathrm{o}}=f_{\Omega} e(x) \mathrm{d} x, e(x) \in \mathscr{S}(x),\right. \\
\text { and } e(x) \text { a compatible strain field }\} .
\end{array}
$$

Above $\int_{\Omega} \cdots=(1 /$ Vol. $\Omega) \int_{\Omega}$ denotes the average over $\Omega$. We will estimate this set in Section 2.3. For now, we simply note that $\mathscr{P}$ is not empty in a SMA, since it always contains $e^{(0)}$ due to self-accommodation.

Once again the situation is as described at the end of Section 2.1 with the set $\mathscr{P}$ replacing $\mathscr{S}$.

\subsection{Bounds on recoverable strain in a polycrystal}

We now estimate the set $\mathscr{P}$ using bounds. The first, and what we will find to be the most useful, bound is the inner bound or Taylor bound. Consider an average strain $e$. Suppose $e$ is recoverable in each grain, i.e. $e \in \mathscr{S}(x)$ for "every" $x \in \Omega$. Then, clearly $e$ is recoverable in the polycrystal or $e \in \mathscr{P}$. Thus

$$
\begin{aligned}
\mathscr{P} & \supset \mathscr{P}_{\mathrm{i}}=\left\{e: R^{\mathrm{T}}(x) e R(x) \in \mathscr{S} \text { for "every" } x\right. \\
& \in \Omega\} .
\end{aligned}
$$

The set $\mathscr{P}_{\mathrm{i}}$ is shown in the shaded region of Fig. 2(a). Geometrically it is the intersection of the set of recoverable strains of the different grains. The set $\mathscr{P}_{\text {i }}$ contains all strains that can be recovered without the cooperative effect of the different grains. Hence, it is a pessimistic or inner bound.

The other bound is the outer bound or Sachs bound. This bound is obtained by allowing each grain to deform as it wishes and forgetting about the constraints they impose on each other. Thus

$$
\begin{aligned}
\mathscr{P} \subset \mathscr{P}_{\mathrm{o}}= & \left\{e: e=f_{\Omega} e(x) \mathrm{d} x, e(x) \in \mathscr{S}(x),\right. \\
& \text { and } e(x) \text { not necessarily compatible }\} .
\end{aligned}
$$

The geometric meaning of this set is harder to visualize. Scale the sets $\mathscr{S}(x)$ for each grain by its own volume fraction. $\mathscr{P}_{\mathrm{o}}$ is the set of all points that can be obtained by adding (vectorically) points in all of these scaled sets as shown in Fig. 2(a). The set $\mathscr{P}_{\text {o }}$ does not account for the constraints between grains; hence, it is an optimistic or outer bound.

It may so happen that the inner and outer bounds are wide apart if $\mathscr{S}$ has small dimension. This is dramatically illustrated in Fig. 2(b) where the set $\mathscr{S}$ is shown schematically as a line. In such a situation, the inner bound reduces to a single point, and we say that the inner bound $\mathscr{P}_{\mathrm{i}}$ is trivial. Bhattacharya and Kohn [32] have argued that the actual set $\mathscr{P}$ is also trivial in such a situation, i.e. $\mathscr{P}=\mathscr{P}_{\text {i }}$ (also see Ref. [53]). In contrast, if $\mathscr{S}$ has high dimension and spans deviatoric strains, the set $\mathscr{P}_{\mathrm{i}}$ is not trivial. In this case, it is generally not true that $\mathscr{P}=\mathscr{P}_{\mathrm{i}}$. However, we believe that even in this case, $\mathscr{P}_{\mathrm{i}}$ is often a good estimate of $\mathscr{P}$, i.e. $\mathscr{P} \cong \mathscr{P}$. We will present examples in support of this claim in Section 3.2. Also see the recent work of Kohn and Niethammer [35].

We close this section by recalling the arguments of Bhattacharya and Kohn [1] on the effect of symmetry. If the change of symmetry is small, such as cubic-tetragonal or cubic-trigonal transformation, the set $\mathscr{S}$ has low dimension; hence $\mathscr{P}_{\text {i }}$ is trivial and the polycrystal has no recoverable strains except for exceptional textures [Fig. 2(b)]. If, on the other hand, the change of symmetry is large, such as cubic-orthorhombic and cubic-monoclinic transformation, the set $\mathscr{S}$ has full dimension. Therefore, the polycrystal has some recoverable strains irrespective of texture [Fig. 2(a)].

\section{MAXIMUM RECOVERABLE STRAIN IN TENSION AND TORSION}

\subsection{Extension or compression}

Consider a polycrystalline SMA below the transformation temperature in the self-accommodated state. Apply a uniaxial stress $\sigma$ to this specimen in the $\xi$ direction. We can show [33] that the maximum recoverable strain $\varepsilon_{\mathrm{R}}$ is given by

$$
\varepsilon_{\mathrm{R}}=\left\{\begin{array}{ll}
\max _{e \in \mathscr{P}}(\xi \cdot e \xi) & \text { if } \sigma>0 \text { (extension) } \\
\min _{e \in \mathscr{P}}(\xi \cdot e \xi) & \text { if } \sigma<0 \text { (compression) }
\end{array} .\right.
$$

The maximum recoverable strain in a single crystal is also given by equation (7) if $\mathscr{P}$ is replaced with $\mathscr{S}$. Equation (7) has an interesting geometrical interpretation. The maximum recoverable strain $\varepsilon_{\mathrm{R}}$ is the maximum projection of the set $\mathscr{P}$ (or $\mathscr{S}$ ) on the loading direction $\xi \otimes \xi$. In single crystals, it is clear from Fig. 1 that the maximum projection of the set $\mathscr{S}$ is always equal to the maximum projection of the transformation strains $e^{(1)}, \ldots, e^{(k)}$. Therefore, it follows that variants coalescence under loading to produce the most favorable variant in a single crystal. Thus, our model coincides with that of Saburi and Nenno [34] except they use a geometrically nonlinear theory.

In polycrystals, we cannot calculate $\varepsilon_{\mathrm{R}}$ since we do not know $\mathscr{P}$. However, we can bound it as 
before using an inner bound $\varepsilon_{\mathrm{R}}^{\mathrm{i}}$ and outer bound $\varepsilon_{\mathrm{R}}^{\mathrm{o}}$. For tensile loading

$$
\varepsilon_{\mathrm{R}}^{\mathrm{i}}=\max _{e \in \mathscr{P}_{\mathrm{i}}}(\xi \cdot e \xi) \text { and } \varepsilon_{\mathrm{R}}^{\mathrm{o}}=\max _{e \in \mathscr{P}_{\mathrm{o}}}(\xi \cdot e \xi) .
$$

It follows from equations (5)-(7) that $\varepsilon_{R}^{\mathrm{i}} \leq \varepsilon_{\mathrm{R}} \leq \varepsilon_{\mathrm{R}}^{\mathrm{o}}$. The inner bound $\varepsilon_{\mathrm{R}}^{\mathrm{i}}$ assumes that every grain undergoes the same strain and it does not take into account the cooperative response between grains. On the other hand, the outer bound $\varepsilon_{\mathrm{R}}^{\mathrm{o}}$ assumes that each grain picks its most favorable variant and ignores the fact that the constraints from the neighboring grains can suppress the most favorable variant. We believe that the inner bound is a reasonable estimate of the actual recoverable strain (see Section 3.2).

Now consider a polycrystal made of $N$ grains with orientations $R_{1}, R_{2}, \ldots, R_{N}$ and volume fraction $\lambda_{1}, \lambda_{2}, \ldots, \lambda_{N}$. We can now write $\varepsilon_{\mathrm{R}}^{\mathrm{i}}$ and $\varepsilon_{\mathrm{R}}^{\mathrm{o}}$ as

$$
\begin{gathered}
\varepsilon_{\mathrm{R}}^{\mathrm{i}}=\max _{R_{j}^{\mathrm{T}} e R_{j} \in \mathscr{S} \forall j=1, \ldots, N}(\xi \cdot e \xi) \\
\varepsilon_{\mathrm{R}}^{\mathrm{o}}=\sum_{j=1}^{N} \max _{e \in \mathscr{S}}\left(\lambda_{j} v_{j} \cdot e v_{j}\right)
\end{gathered}
$$

where $v_{j}=R_{j}^{\mathrm{T}} \xi$. We can interpret equations (9) and (10) geometrically as shown in Fig. 2(a): the inner bound is the maximum projection of the set $\mathscr{P}_{\mathrm{i}}$ on the loading direction while the outer bound is the average of the maximum projection of each set $R_{j} \mathscr{S} R_{j}^{\mathrm{T}}$ on the loading direction weighted by its volume fraction $\lambda_{j}$. Note also from Fig. 2(a) that $\varepsilon_{\mathrm{R}}^{\mathrm{i}}$ can be strictly smaller than the smallest recoverable strain amongst all grains.

We can rewrite equations (9) and (10) as linear programming problems if $\mathscr{S}$ is the convex hull of stress-free variants. The inner bound

$$
\varepsilon_{\mathrm{R}}^{\mathrm{i}}=\max _{\mu_{i}^{j}}\left(\sum_{i=1}^{k} \xi \cdot\left(\mu_{i}^{1} R_{1} e^{(i)} R_{1}^{\mathrm{T}}\right) \xi\right)
$$

where the maximum is taken over the following constraints:

$$
\begin{gathered}
\sum_{i=1}^{k} \mu_{i}^{j} R_{j} e^{(i)} R_{j}^{\mathrm{T}}=\sum_{i=1}^{k} \mu_{i}^{1} R_{1} e^{(i)} R_{1}^{\mathrm{T}} \quad \text { for } j=2, \ldots, N \\
\sum_{i=1}^{k} \mu_{i}^{j}=1, \mu_{i}^{j} \geq 0 \quad \text { for } j=1, \ldots, N
\end{gathered}
$$

and the outer bound

$$
\varepsilon_{\mathrm{R}}^{\mathrm{o}}=\sum_{j=1}^{N} \lambda_{j}\left(\max _{\mu_{i} \geq 0, \Sigma_{i=1}^{k} \mu_{i}=1} \xi \cdot\left(\sum_{i=1}^{k} \mu_{i} R_{j} e^{(i)} R_{j}^{\mathrm{T}}\right) \xi\right) .
$$

Recall that in the cubic-monoclinic transformation $\mathscr{S}_{\text {mono }}$ is unknown. Therefore, we cannot evaluate equation (9). Instead, we use

$$
\begin{gathered}
\varepsilon_{\mathrm{R}}^{\mathrm{i}}=\max _{R_{j}^{\mathrm{T}} e R_{j} \in \mathscr{S}_{\text {orth }} \forall j=1, \ldots, N}(\xi \cdot e \xi) \\
\varepsilon_{\mathrm{R}}^{\mathrm{e}}=\max _{R_{j}^{\mathrm{T}} e R_{j} \in \mathscr{S}_{\text {mono }}^{\mathrm{c}} \forall j=1, \ldots, N}(\xi \cdot e \xi) \\
\varepsilon_{\mathrm{R}}^{\mathrm{o}}=\sum_{j=1}^{N} \max _{e \in \mathscr{S}_{\text {mono }}^{\mathrm{c}}\left(\lambda_{j} v_{j} \cdot e v_{j}\right)}
\end{gathered}
$$

as the inner bound, inner estimate and outer bound, respectively. We can rewrite equations (14)(16) as linear programming problems as before.

\subsection{Maximum recoverable strain in a laminated polycrystal}

We have claimed above that the inner bound provides a reasonable estimate to the actual recoverable strain. This is justified with an example - a polycrystal with a laminated texture. Laminated microstructures have been used extensively in the study of polycrystals and composite materials since the exact effective properties can be obtained by solving an algebraic problem (see, e.g. Ref. [54]). We consider the orthorhombic martensite where we know the exact set $\mathscr{S}_{\text {orth }}$. A rank-two laminate containing two orientations $R_{1}$ and $R_{2}$ is shown in Fig. 3. Here, $n_{1}, n_{2}$ and $n_{3}$ are the normals of the laminates with the corresponding volume fraction $\lambda_{1}, \lambda_{2}$ and $\lambda_{3}$, respectively.

If we assume a wide separation of scales between the two levels of the laminate, it can be shown that the strain is piecewise constant with values $e_{1}, e_{2}, e_{3}$ and $e_{4}$ as also shown in Fig. 3. Kinematic compatibility or coherence forces the following constraints:

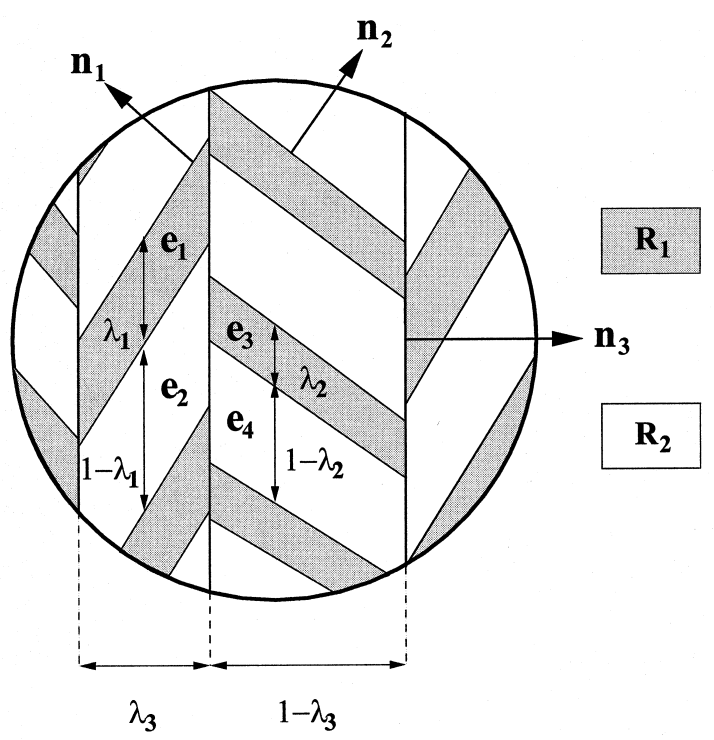

Fig. 3. A rank-two laminate containing two orientations $R_{1}$ and $R_{2}$. 
Table 3. Orientation, normals and volume fraction of the rank-two laminates used in the calculation for $\mathrm{Ti}-40 \mathrm{Ni}-10 \mathrm{Cu}(\mathrm{B} 19)$ and $\gamma_{1}^{\prime} \mathrm{Cu}-$ $14 \mathrm{Al}-4 \mathrm{Ni}(\mathrm{wt} \%)$

\begin{tabular}{|c|c|c|c|c|c|c|c|c|c|c|}
\hline \multirow[t]{2}{*}{ SMA } & \multicolumn{4}{|c|}{ Orientation } & \multicolumn{3}{|c|}{ Normals } & \multicolumn{3}{|c|}{ Volume fraction } \\
\hline & & & $R_{2}$ & & $n_{1}$ & $n_{2}$ & $n_{3}$ & $\lambda_{1}$ & $\lambda_{2}$ & $\lambda_{3}$ \\
\hline $\mathrm{Ti}-40 \mathrm{Ni}-10 \mathrm{Cu}$ & $\left.\begin{array}{c}0 \\
0 \\
\sqrt{2}\end{array}\right)$ & $\frac{1}{\sqrt{6}}\left(\begin{array}{l}\sqrt{3} \\
\sqrt{2} \\
-1\end{array}\right.$ & $\begin{array}{l}-\sqrt{3} \\
\sqrt{2} \\
-1\end{array}$ & $\left.\begin{array}{c}0 \\
\sqrt{2} \\
2\end{array}\right)$ & & & $\left(\begin{array}{l}1 \\
0\end{array}\right)$ & 0.5 & 0.5 & 0.5 \\
\hline $\mathrm{Cu}-14 \mathrm{Al}-4 \mathrm{Ni}$ & $\left.\begin{array}{cc}1 & 0 \\
& \sqrt{2} \\
1 & 0\end{array}\right)$ & $\frac{1}{\sqrt{6}}\left(\begin{array}{c}\sqrt{3} \\
1\end{array}\right.$ & $\begin{array}{c}-\sqrt{3} \\
1 \\
-\sqrt{2}\end{array}$ & $\left.\begin{array}{c}0 \\
2 \\
\sqrt{2}\end{array}\right)$ & $\left(\begin{array}{l}0 \\
0 \\
1\end{array}\right)$ & & $\left(\begin{array}{l}1 \\
0\end{array}\right.$ & 0.5 & 0.5 & 0.5 \\
\hline
\end{tabular}

$e_{1}-e_{2}=\frac{1}{2}\left(n_{1} \otimes m_{1}+m_{1} \otimes n_{1}\right)$,

$$
\begin{aligned}
& e_{3}-e_{4}=\frac{1}{2}\left(n_{2} \otimes m_{2}+m_{2} \otimes n_{2}\right), \\
& \left(\lambda_{1} e_{1}+\left(1-\lambda_{1}\right) e_{2}\right)-\left(\lambda_{2} e_{3}+\left(1-\lambda_{2}\right) e_{4}\right) \\
= & \frac{1}{2}\left(n_{3} \otimes m_{3}+m_{3} \otimes n_{3}\right)
\end{aligned}
$$

for some vectors $m_{1}, m_{2}$ and $m_{3}$ and average strain is

$$
\begin{aligned}
e_{\mathrm{ave}}= & \lambda_{3}\left(\lambda_{1} e_{1}+\left(1-\lambda_{1}\right) e_{2}\right) \\
& +\left(1-\lambda_{3}\right)\left(\lambda_{2} e_{3}+\left(1-\lambda_{2}\right) e_{4}\right) .
\end{aligned}
$$

Moreover, to be recoverable, $e_{1}, e_{2}, e_{3}$ and $e_{4}$ must satisfy

$$
R_{1}^{\mathrm{T}} e_{1} R_{1}, R_{2}^{\mathrm{T}} e_{2} R_{2}, R_{1}^{\mathrm{T}} e_{3} R_{1}, R_{2}^{\mathrm{T}} e_{4} R_{2} \in \mathscr{S}_{\text {orth }} .
$$

Putting all these in equation (7), we can conclude that for tensile loading in the $\xi$ direction, the maximum recoverable strain is given by

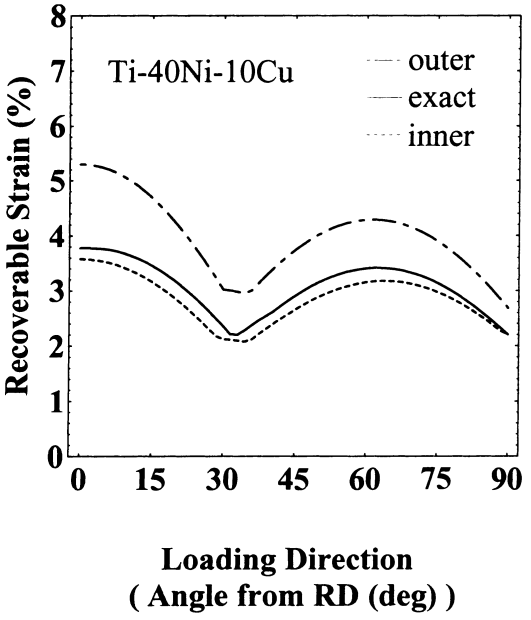

(a)

$$
\varepsilon_{\mathrm{R}}=\max \xi \cdot e_{\mathrm{ave}} \xi
$$

where the maximum is taken over $e_{1}, e_{2}, e_{3}$ and $e_{4}$ subject to constraints (17) and (18).

Let us consider two examples: $\mathrm{Ti}-40 \mathrm{Ni}-10 \mathrm{Cu}$ (B19) and $\gamma_{1}^{\prime} \mathrm{Cu}-14 \mathrm{Al}-4 \mathrm{Ni}$ (wt\%), both undergoing cubic-orthorhombic transformation. The texture, normals and volume fraction of the rank-two laminate are listed in Table 3 and the tensile loading direction for both cases is $\xi=(\cos \theta, 0, \sin \theta)^{\mathrm{T}}$ for various $\theta$. In fact, we will find later that this is exactly like the rolled sheet subjected to tensile loading along the rolling plane (see Section 5 and Fig. 6). Figure 4 shows the inner bound, the outer bound and the exact value of the maximum recoverable strain calculated according to equations (9), (10) and (20), respectively. We have repeated the same calculations for different laminates, i.e. for different normals of $n_{1}, n_{2}$ and $n_{3}$. The results are either the same or closer to the inner bound except in a very few exceptional situations. We have also performed the calculations for a rank-three laminate. The results are even closer to the inner bound.

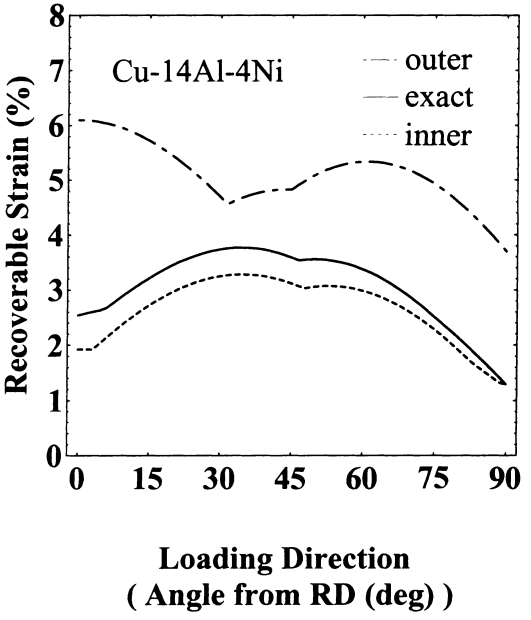

(b)

Fig. 4. The inner bound, outer bound and exact value of the maximum recoverable strain for the ranktwo laminated polycrystal subjected to uniaxial tension in different directions: (a) $\mathrm{Ti}-40 \mathrm{Ni}-10 \mathrm{Cu}(\mathrm{B} 19)$; (b) $\gamma_{1}^{\prime} \mathrm{Cu}-14 \mathrm{Al}-4 \mathrm{Ni}(\mathrm{wt} \%)$. 
It is clear that the constraints between the grains is overwhelming and hence $\varepsilon_{\mathrm{R}} \cong \varepsilon_{\mathrm{R}}^{\mathrm{i}}$. Finally, the drawback of the outer bound can also be seen especially in the case of $\gamma_{1}^{\prime} \mathrm{Cu}-14 \mathrm{Al}-4 \mathrm{Ni}(\mathrm{wt} \%)$ at the loading direction $\theta=0^{\circ}$.

To understand the effect of the intergranular constraint, we show in Fig. 5 the volume fractions of the different orthorhombic variants calculated according to the exact solution as well as the inner and outer bounds in the grains with orientation $R_{2}$ for the case $\theta=60^{\circ}$. Variant 6 is the most favorable variant in both $\mathrm{Ti}-\mathrm{Ni}-\mathrm{Cu}$ and $\mathrm{Cu}-\mathrm{Al}-\mathrm{Ni}$ for this orientation and hence the outer bound uses $100 \%$ variant 6 . However, compatibility forces variants $1,3,4$ and 5 to also form in $\mathrm{Ti}-\mathrm{Ni}-\mathrm{Cu}$ and variants 1 and 3 to also form in $\mathrm{Cu}-\mathrm{Al}-\mathrm{Ni}$ in the exact solution. The inner bound which respects compatibility does not get the volume fractions exactly correct, but is close.

\subsection{Combined tension-torsion}

Consider a shape-memory wire or a circular tube below the transformation temperature in the selfaccommodated state. Pull it uniaxially to the given strain $\varepsilon_{0}$ and apply a torque $M$ to the ends. We wish to find $w_{\mathrm{R}}$, the maximum recoverable twist (angle per unit length). Notice that $w_{R}$ is not dimensionless; it depends on the outer radius $r_{0}$ of the specimen. However, $r_{0} w_{\mathrm{R}}$ is dimensionless and is independent of the dimensions of the specimen. Therefore, we estimate the maximum recoverable normalized twist $\gamma_{\mathrm{R}}=r_{0} w_{\mathrm{R}}$. It will be clear that the normalized twist $\gamma_{R}$ is also the shear strain in the outer-most part of the cross section.

Torsion is a difficult problem in general anisotropic bodies. Therefore, we assume that the grains are very small and the specimen is macroscopically uniaxial in symmetry. Choose any coordinate system with $i_{3}$ the axis of the wire or tube and define

$$
e\left(\gamma, \theta, \alpha_{i}\right)=\left(\begin{array}{ccc}
\alpha_{1} & \alpha_{2} & -\gamma \cos \theta \\
\alpha_{2} & \alpha_{3} & \gamma \sin \theta \\
-\gamma \cos \theta & \gamma \sin \theta & \varepsilon_{0}
\end{array}\right) .
$$

Then, it is possible to show [33] that the maximum recoverable normalized twist

$$
\gamma_{\mathrm{R}}=\max _{\alpha_{i}} \max _{e\left(\gamma, \theta, \alpha_{i}\right) \in \mathscr{P} \forall \theta} \gamma .
$$

Equation (22) cannot be solved without knowing the set $\mathscr{P}$. However, we can bound it by replacing $\mathscr{P}$ with $\mathscr{P}_{\mathrm{i}}$ and $\mathscr{P}_{\mathrm{o}}$ to get the inner and outer bound, respectively. In particular, in the case of materials undergoing cubic-monoclinic transformation, we obtain the inner bound $\gamma_{\mathrm{R}}^{\mathrm{i}}$, inner estimate $\gamma_{\mathrm{R}}^{\mathrm{e}}$ and outer bound $\gamma_{\mathrm{R}}^{\mathrm{o}}$ analogously to equations (14)(16), respectively.

\section{THIN FILMS}

We now extend the results of Sections 2 and 3 to polycrystalline thin films. It is well known that microstructure can be significantly different in thin films as compared to bulk materials. Recently Bhattacharya and James [41] have developed a theory of single crystal martensitic thin films which captures this effect. It is possible to extend this theory to polycrystalline thin films following methods similar to Sections 2 and 3 if the texture satisfies certain conditions: the grains are columnar (i.e. they extend through the thickness) and the film normal is crystallographically the same in each grain. If the texture does not satisfy this condition, the theory becomes more complicated. Fortunately, sputtered films do satisfy this condition and we limit ourselves to this case. Here we present the main ideas and summarize the results referring the reader to Shu $[33,55]$ for details. We note in particular that grain size changes as we go from bulk to sputtered films and this has important conse-
Ti-40Ni-10Cu

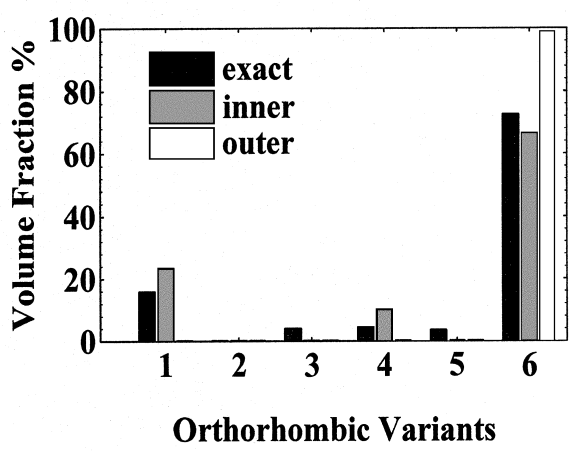

(a)

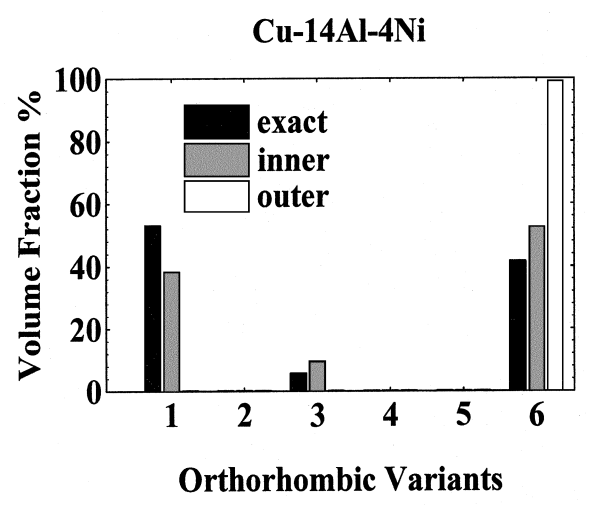

(b)

Fig. 5. Constraints from neighboring grains can suppress the most favorably oriented variant. Volume fractions of the different orthorhombic variants in the grains with orientation $R_{2}$ for the case $\theta=60^{\circ}$ calculated according to the exact solution as well as inner and outer bounds: (a) $\mathrm{Ti}-40 \mathrm{Ni}-10 \mathrm{Cu}$ (B19); (b) $\gamma_{1}^{\prime} \mathrm{Cu}-14 \mathrm{Al}-4 \mathrm{Ni}(\mathrm{wt} \%)$. 
quences. This will not be discussed here to keep the length of this paper manageable, but in a forthcoming paper [55] which addresses in detail the influence of length scales of microstructure, film thickness and grain size on the limiting behavior of heterogeneous thin films.

The main difference in the microstructure between bulk materials and thin films can be explained in terms of kinematics: as the film becomes very thin, only the in-plane components of the strain need to be compatible. Consider a film with normal $f$ and let $\left\{f_{1}, f_{2}, f_{3}\right\}$ be an orthonormal basis so that $f_{1}$ and $f_{2}$ lie in the plane of the film. A strain field $e(x)$ has to satisfy only

$$
\frac{\partial^{2} e_{11}}{\partial x_{2}^{2}}+\frac{\partial^{2} e_{22}}{\partial x_{1}^{2}}=2 \frac{\partial^{2} e_{12}}{\partial x_{1} \partial x_{2}}
$$

but not the other five compatibility conditions. Alternatively, consider an interface $\Gamma$ between two strains $e_{1}$ and $e_{2}$. Compatibility in thin films requires

$$
e_{1}-e_{2}=\frac{1}{2}(a \otimes n+n \otimes a)+\frac{1}{2}(c \otimes f+f \otimes c)
$$

for some vectors $a$ and $c$, and where $n$ is the normal to the interface in the plane of the film. Contrast this with bulk materials where compatibility requires equation (1) which is equation (24) with $c=0$. The term $\frac{1}{2}(c \otimes f+f \otimes c)$ represents incompatibility in the thickness direction. This can be overcome by a local strain field or transition layer whose energy goes to zero with thickness. Therefore, the compatibility or coherence requirement is weakened in thin films and this allows a variety of deformations like the paper-folding deformations, tunnels and tents, and exact austenite/martensite interfaces in a single crystal film [41].

As before, we can define the set of recoverable strains $\mathscr{S}^{f}$ in a single crystal thin film as the set of average strains of microstructures of martensitic variants. Since the thin-film compatibility or coherence conditions (23) and (24) depend on the film normal, $\mathscr{S}^{f}$ also depends on it. We can show that all pairs of monoclinic variants are pair-wise compatible for $\{100\}$ and $\{110\}$ films in $\mathrm{Ti}-\mathrm{Ni}$ and $\{110\}$ and $\{111\}$ films in $\mathrm{Cu}-\mathrm{Zn}-\mathrm{Al}$. Here, $\{h k l\}$ denotes the thin film normal $f$ in the cubic basis. Thus, for these films, $\mathscr{S}^{f}=\mathscr{S}_{\text {mono }}^{\mathrm{c}}$. In contrast, not all pairs are compatible for $\{111\}$ films in $\mathrm{Ti}-\mathrm{Ni}$ and $\{100\}$ films in $\mathrm{Cu}-\mathrm{Zn}-\mathrm{Al}$. In those cases, the set $\mathscr{S}^{f}$ becomes more complicated [33].

We now turn to polycrystalline thin films. We assume that the grains are columnar and the film normal is crystallographically identical in each grain, $R$ is a rotation about the film normal and is a function only of the in-plane coordinates. Further, we assume the following separation of length scales: film thickness and microstructure are smaller than the lateral diameter of each grain which in turn is smaller than the specimen. Then, we can follow the arguments of Sections 2 and 3 to conclude that the set of recoverable strains in a polycrystalline thin film with normal $f$ is

$$
\begin{aligned}
\mathscr{P}^{f}= & \left\{\langle e(x)\rangle: e(x) \in R(x) \mathscr{S}^{f} R^{\mathrm{T}}(x)\right. \\
& \text { and } \left.\frac{\partial^{2} e_{11}}{\partial x_{2}^{2}}+\frac{\partial^{2} e_{22}}{\partial x_{1}^{2}}=2 \frac{\partial^{2} e_{12}}{\partial x_{1} \partial x_{2}}\right\}
\end{aligned}
$$

where $\langle e(x)\rangle$ denotes the average over the entire film. The corresponding inner and outer bounds are

$$
\begin{aligned}
\mathscr{P}_{\mathrm{i}}^{f} & =\{\langle e(x)\rangle: e(x) \\
& \left.\in R(x) \mathscr{S}^{f} R^{\mathrm{T}}(x), e_{11}, e_{12} \text { and } e_{22} \text { are constants }\right\}
\end{aligned}
$$

$$
\mathscr{P}{ }_{\mathrm{o}}^{f}=\mathscr{P}_{\mathrm{o}} .
$$

Finally, when subjected to uniaxial in-plane tension in the $\xi$ direction, the inner bound on the maximum recoverable extension is

$$
\varepsilon_{\mathrm{R}}^{\mathrm{i} f}=\max _{e \in \mathscr{P}_{i}^{f}}(\xi \cdot e \xi) .
$$

\section{RESULTS FOR UNIAXIAL LOADING}

We now evaluate the theoretical bounds on recoverable strains under uniaxial loading for specific textures. Experimentally observed textures will be used for comparison, and idealized textures for prediction. We focus on $\mathrm{Ti}-\mathrm{Ni}$ and $\mathrm{Cu}-\mathrm{Zn}-\mathrm{Al}$ alloys. The results for other Cu-based SMAs are very similar to those of $\mathrm{Cu}-\mathrm{Zn}-\mathrm{Al}$.

\subsection{Rolling texture: comparison with experimental observations}

Zhao and Beyer $[20,21]$ have recently measured the texture and recoverable strain in rolled $\mathrm{Ti}-\mathrm{Ni}-$ $\mathrm{Cu}$ sheets. After rolling, the specimens were heat treated at a temperature below the recrystallization temperature of this alloy. The $\mathrm{Cu}$ composition was around $5 \%$ so that the martensite phase was monoclinic similar to $\mathrm{Ti}-\mathrm{Ni}[46,56]$. The observed texture is shown in Fig. 6: $60 \%$ of the grains had a $\{110\}\langle 110\rangle$ orientation in the parent phase while $40 \%$ of the grains had a $\{111\}\langle 110\rangle$ orientation. Here, $\{h k l\}\langle u v w\rangle$ means that $\{h k l\}$ planes lie parallel to the rolling plane and $\langle u v w\rangle$ directions lie parallel to the rolling direction (RD). ND and TD denote the normal and transverse directions of the rolled sheet. Specimens were cut along different loading directions (LDs) along the plane of the sheet and the recoverable strains were measured by the thermal cycling tensile test. The experimental results are shown as dark circles $\bullet$ in Fig. 7. 


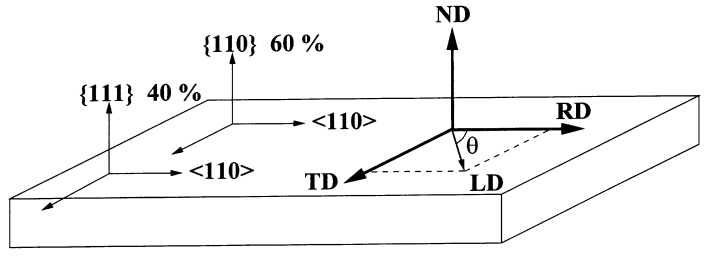

Fig. 6. Directions in a rolled sheet and texture observed by Zhao and Beyer.

Theoretical bounds computed using equations (14)-(16) are shown in Fig. 7(a). Here, the upper dashed line $(\mathrm{OB})$ is the outer bound $\varepsilon_{\mathrm{R}}^{\mathrm{o}}$, the dashed line in the middle (IE) is the inner estimate $\varepsilon_{\mathrm{R}}^{\mathrm{e}}$ and the thin continuous line (IB) is the inner bound $\varepsilon_{\mathrm{R}}^{\mathrm{i}}$. As expected, the experimental observations of recoverable strains lie close to the inner estimate and inner bound while the outer bound is a serious overestimate.

However, the experimental result near the RD violates the inner bound. There are a variety of reasons. Among them is the fact that the texture is not ideal; not all grains have the $\{111\}\langle 110\rangle$ or $\{110\}\langle 110\rangle$ orientation, but contain certain "wobble" about the ideal orientation. Therefore, we take 100 grains with the following orientation: 40 grains are oriented randomly within $5^{\circ}$ of $\{111\}\langle 110\rangle$ while 60 grains are oriented randomly within $5^{\circ}$ of $\{110\}\langle 110\rangle$. We call this a texture with $5^{\circ}$ wobble. The results are shown in Fig. 7(b). We have also repeated the calculation using 200 grains with the same distribution and find the results are essentially identical. However, if the wobble is taken as $10^{\circ}$ instead of $5^{\circ}[18,57]$, the inner estimate (IE) and inner bound (IB) drop about $1 \%$ in the vicinity of $\mathrm{RD}$ and remain unchanged near the TD.

We also compare our results with similar experiments of Inoue et al. [18]. First consider their PLCR specimens which have a strong $\{111\}$ fiber \| ND. This means the rolling planes are $\{111\}$ in the parent phase while the RD is random. Our bounds calculated with $5^{\circ}$ wobble and their experimental observations are shown in Fig. 8(a). As expected, the results are almost isotropic in the plane and the experimental results are close to the inner bound and the inner estimate. Next, consider their PL-RX specimens which contain $\langle 110\rangle$ partial fiber $\|$ RD with peaks at $\{332\}\langle 110\rangle$ and $\{111\}\langle 110\rangle$ accompanied with a spread towards $\{110\}\langle 110\rangle$. This texture is similar to that observed by Zhao and Beyer $[20,21]$ except that the distribution of the rolling planes is different. Our bounds calculated with $5^{\circ}$ wobble are compared with their experimental observations in Fig. 8(b).

The inner bounds and estimates, which we emphasize do not use any adjustable parameters, are in general agreement with the observed recoverable strains: they pick out the level and the trend. However, they do differ in one crucial aspect. In particular, notice in Figs 7 and 8(b) that the inner bound contains a cusp-like dip around $35 \mathrm{deg}$. The main reason, we believe, is the fact that for a monoclinic material we use the set $\mathscr{S}_{\text {orth }}$ instead of $\mathscr{S}_{\text {mono }}$ to calculate the inner bound. The difference between these sets is significant in some isolated directions; and one such direction corresponds to the cusp-like dip for these textures. This is indeed a weakness of our inner bound which uses $\mathscr{S}_{\text {orth }}$, and calculation of the set $\mathscr{S}_{\text {mono }}$ still remains a major outstanding problem. It is to overcome this weak-

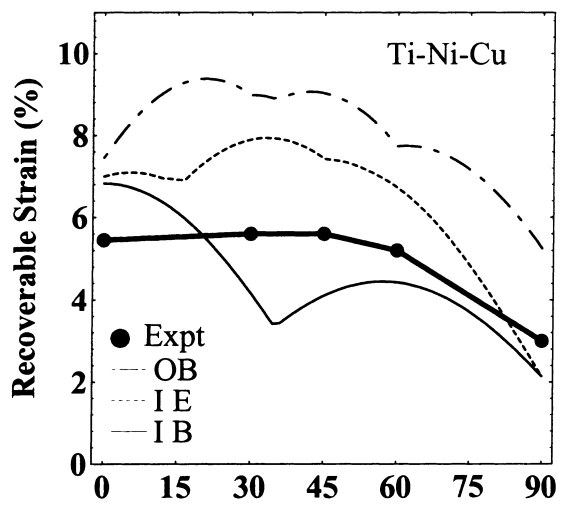

Loading Direction ( Angle from RD (deg) )

(a)

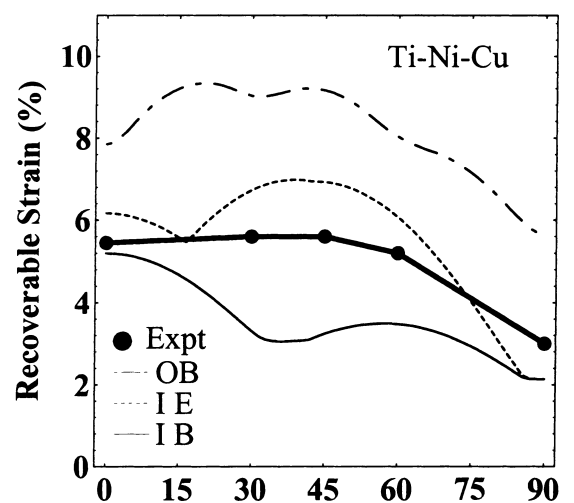

Loading Direction ( Angle from RD (deg) )

(b)

Fig. 7. Comparison of theory and experiment for the Zhao-Beyer texture. Here, $\bullet$ Expt is the experimentally measured recoverable strain. OB, IE and IB mean outer bound $\varepsilon_{\mathrm{R}}^{\mathrm{o}}$, inner estimate $\varepsilon_{\mathrm{R}}^{\mathrm{e}}$ and inner bound $\varepsilon_{\mathrm{R}}^{\mathrm{i}}$, respectively: (a) ideal texture; (b) texture with $5^{\circ}$ wobble. 


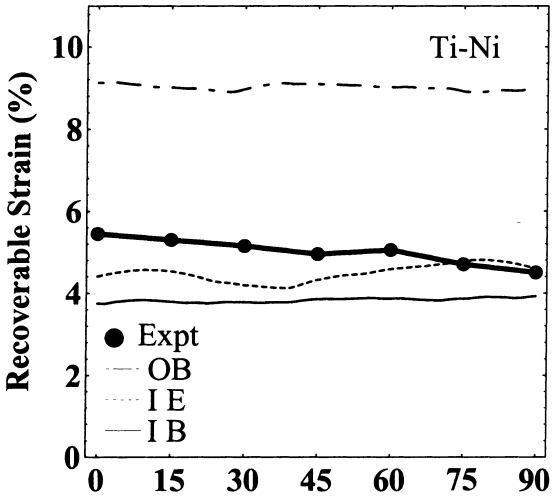

Loading Direction ( Angle from RD (deg) )

(a)

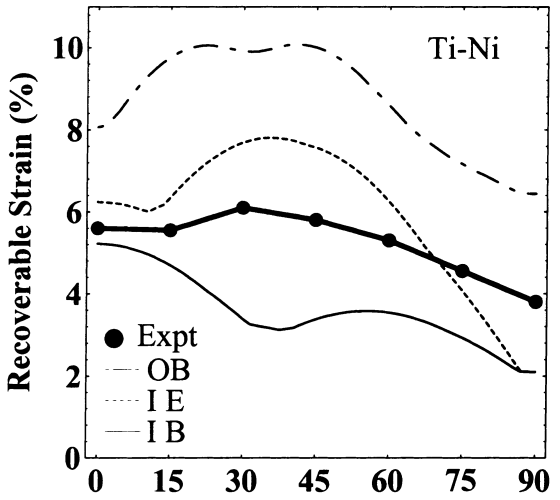

\section{Loading Direction} ( Angle from RD (deg) )

(b)

Fig. 8. Comparison of theory and experiment for the Inoue-Miwa-Inakazu textures. Here, • Expt is the measured recoverable strain. OB, IE and IB mean outer bound $\varepsilon_{\mathrm{R}}^{\mathrm{o}}$, inner estimate $\varepsilon_{\mathrm{R}}^{\mathrm{e}}$ and inner bound $\varepsilon_{\mathrm{R}}^{\mathrm{i}}$, respectively: (a) PL-CR specimens with $\{111\}\langle u v w\rangle$ texture; (b) PL-RX specimens with $\langle 110\rangle$ partial fiber with components from $\{111\}\langle 110\rangle$ to $\{110\}\langle 110\rangle$.

ness that we also include the inner estimate. A significant difference between the inner bound and the estimate in some loading direction provides a warning that the inner bound may be too conservative in this direction. $\dagger$ A second reason for the deviation is the fact that we use only the important fiber components (with wobble) rather than the exact measured texture.

\subsection{Rolling texture: prediction on fiber components}

Most SMAs including $\mathrm{Ti}-\mathrm{Ni}$ and $\mathrm{Cu}-\mathrm{Zn}-\mathrm{Al}$ have a body-centered-cubic (b.c.c.) superlattice structure in the austenite phase [58]. Common b.c.c. textures are listed in Table 4 [59-62]. We note that $\alpha$-fiber II is not common in general, but is observed in SMAs [18, 20, 21].

Figure 9 and 10 show the inner bound and estimate for $\mathrm{Ti}-\mathrm{Ni}$ and $\mathrm{Cu}-\mathrm{Zn}-\mathrm{Al}$ for the different textures listed in Table 4. Comparing these, it is clear that the recoverable strains are much higher in $\mathrm{Ti}-$ $\mathrm{Ni}$ than in $\mathrm{Cu}-\mathrm{Zn}-\mathrm{Al}$ for typical b.c.c. textures. The $\alpha$-fiber I in Ti-Ni exhibits two peaks in RD and TD of the rolled sheet as shown in Fig. 9(a). Therefore, this texture is desirable for uniaxial applications since it is easy to extract good specimens from a rolled sheet. On the other hand, the $\gamma$-fiber observed in Ref. [18] exhibits the least anisotropy as seen in Fig. 9(c). This texture is preferred if the recoverable strain is required in every direction of the rolled sheet. In Cu-based SMAs, the shaperecovery strain while poor in general is expected to

$\dagger$ At the same time, close agreement between the inner bound and estimate (as in Fig. 8(a)) indicates that these are reliable predictions. be relatively large for the $\eta$-fiber. Unfortunately, this fiber is usually less common. We also note that for these alloys, the behavior of $\alpha$-fiber I can be improved by restricting the fiber components from $\{001\}\langle 110\rangle$ to $\{112\}\langle 110\rangle$ : the result is similar to that of Fig. 10(a) except the peak of the inner estimate rises to about $5 \%$ around $45^{\circ}$. Park and Bunge [24-26] have observed this texture in a hotrolled $\mathrm{Cu}-\mathrm{Zn}-\mathrm{Al}$ sheet although no recoverable strain measurements are available.

\subsection{Other textures}

5.3.1. Random texture. The inner bound and inner estimate on the maximum recoverable extension for a polycrystal with randomly oriented grains are shown in Table 5 for $\mathrm{Ti}-\mathrm{Ni}$ and $\mathrm{Cu}-\mathrm{Zn}-\mathrm{Al}$. The behavior of these alloys is quite similar, and rather poor with random texture.

5.3.2. Wire drawing texture. Body-centered-cubic metals and alloys have been shown to develop strong $\langle 110\rangle$ fiber texture after drawing or extrusion [63]. Assuming equal volume fraction in all of the fiber components, the inner bound and estimate of tensile recoverable strain in $\mathrm{Ti}-\mathrm{Ni}$ and $\mathrm{Cu}-\mathrm{Zn}-\mathrm{Al}$ are listed in Table 5. Clearly, Ti-Ni is

Table 4. Some common texture fibers observed in b.c.c. metals and alloys. The $\alpha$-fiber II is the characteristic texture of Ti-Ni with the B2-lattice

\begin{tabular}{lcc}
\hline Fiber & \multicolumn{2}{c}{ Fiber axis Relevant components associated with the fiber } \\
\hline$\alpha$-fiber I & $\langle 110\rangle \| \mathrm{RD}$ & $\{001\}\langle 110\rangle-\{112\}\langle 110\rangle-\{111\}\langle 110\rangle$ \\
$\alpha$-fiber II & $\langle 110\rangle \| \mathrm{RD}$ & $\{111\}\langle 110\rangle-\{110\}\langle 110\rangle$ \\
$\gamma$-fiber & $\{111\} \| \mathrm{ND}$ & $\{111\}\langle 110\rangle-\{111\}\langle 112\rangle$ \\
$\eta$-fiber & $\langle 100\rangle \| \mathrm{RD}$ & $\{001\}\langle 100\rangle-\{011\}\langle 100\rangle$ \\
\hline
\end{tabular}




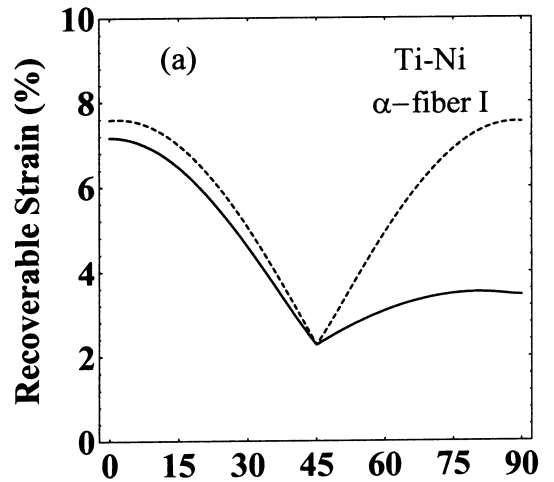

Loading Direction ( Angle from RD (deg) )

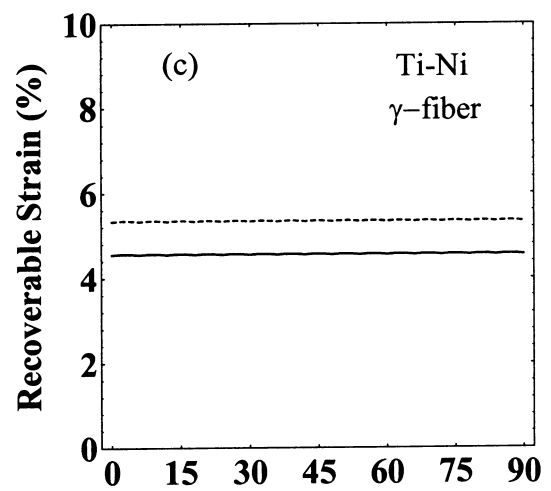

\section{Loading Direction ( Angle from RD (deg) )}

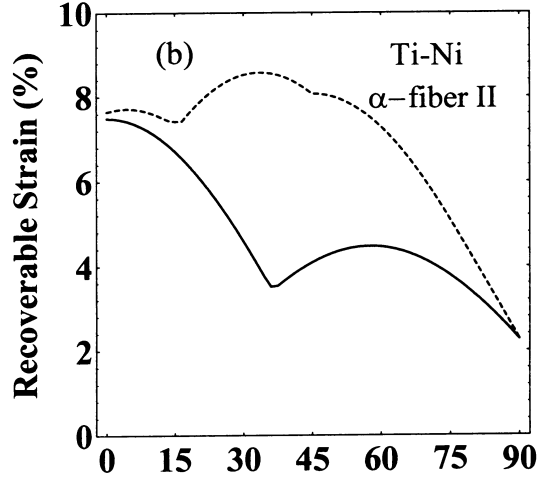

Loading Direction ( Angle from RD (deg) )

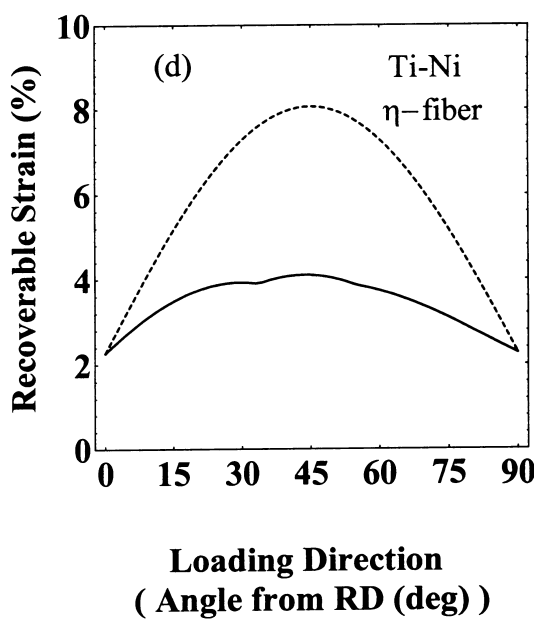

Fig. 9. The predicted uniaxial recoverable extension in $\mathrm{Ti}-\mathrm{Ni}$ for various rolling textures. The upper dashed line is the inner estimate $\varepsilon_{\mathrm{R}}^{\mathrm{e}}$ while the lower continuous line is the inner bound $\varepsilon_{\mathrm{R}}^{\mathrm{i}}$.

expected to have much better shape-memory behavior than $\mathrm{Cu}-\mathrm{Zn}-\mathrm{Al}$ for this fiber. Park et al. [27] have observed a strong $\langle 110\rangle$ fiber texture in the parent phase in cold drawn $\mathrm{Cu}-\mathrm{Zn}-\mathrm{Al}$ wires although no recoverable strain measurements are available.

Yamauchi et al. [22] observed a $\langle 111\rangle$ recrystallization fiber texture in cold-drawn and subsequently annealed $\mathrm{Ti}-\mathrm{Ni}$ rods. Similar observation was reported in Ref. [23] for $\mathrm{Ti}-\mathrm{Ni}-\mathrm{Cu}$ wire. Our bounds for this $\langle 111\rangle$ texture are also listed in Table 5. Again, this texture is more favorable in $\mathrm{Ti}-\mathrm{Ni}$ than in $\mathrm{Cu}-\mathrm{Zn}-\mathrm{Al}$.

Finally, Miyazaki et al. [12] have reported that drawn wires of $\mathrm{Ti}-50.6 \mathrm{Ni}$ alloy can recover around $6 \%$ tensile strain although there is no additional information to show those wires have such $\langle 110\rangle$ or $\langle 111\rangle$ fiber texture.

5.3.3. Solidification texture. Eucken and coworkers $[8,28,29]$ have obtained a columnar grain structure with a strong $\{100\}$ texture in meltspun ribbons of both $\mathrm{Ti}-\mathrm{Ni}$ alloy and $\mathrm{Cu}$-based SMAs. Their experimental results show that $\mathrm{Ti}-\mathrm{Ni}$ ribbon can recover less than $4 \%$ tensile strain, but $\mathrm{Cu}-\mathrm{Al}-$ $\mathrm{Ni}$ ribbon can recover as large as $6.5 \%$ strain. Indeed, this difference can be predicted using the inner bound [1]. Clearly, $\{100\}$ texture favors larger recoverable strain in $\mathrm{Cu}$-based $\mathrm{SMAs}$ than in $\mathrm{Ti}-\mathrm{Ni}$ alloys.

5.3.4. Sputtering texture. Thin films of the shapememory material $\mathrm{Ti}-\mathrm{Ni}$ and closely related alloys have been made by magnetron sputtering by several groups (Refs [37-40] and references there). This method produces polycrystalline films with special texture: the grains are columnar and the film normal is crystallographically identical in each grain. In b.c.c.-based materials, including $\mathrm{Ti}-\mathrm{Ni}$ and $\mathrm{Cu}-\mathrm{Zn}-\mathrm{Al}$, the film normal is typically $\{110\}[31,64,65]$. The inner bound and estimate on the in-plane recoverable strain are shown in Table 5. For relatively thick films, one should use the inner bound $\varepsilon_{\mathrm{R}}^{\mathrm{i}}$ and the inner estimate $\varepsilon_{\mathrm{R}}^{\mathrm{e}}$. For 


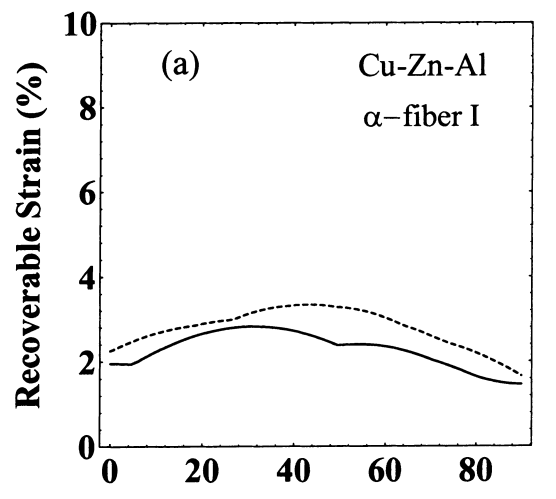

Loading Direction ( Angle from RD (deg) )

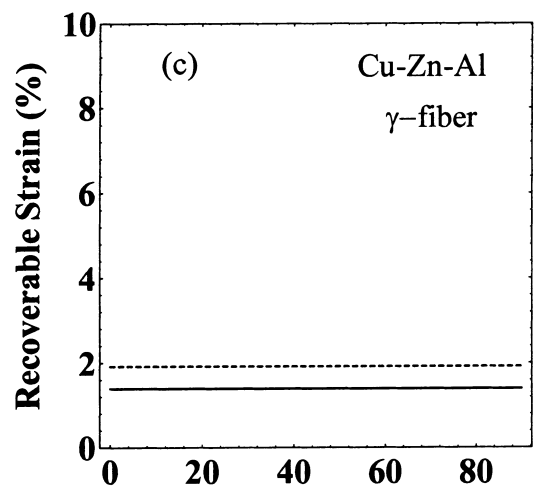

Loading Direction ( Angle from RD (deg) )

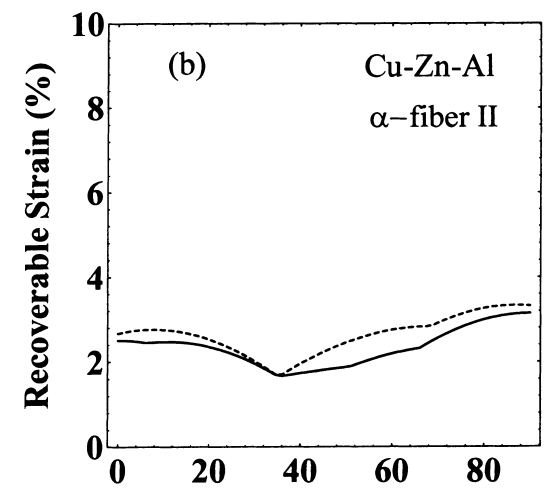

Loading Direction ( Angle from RD (deg) )

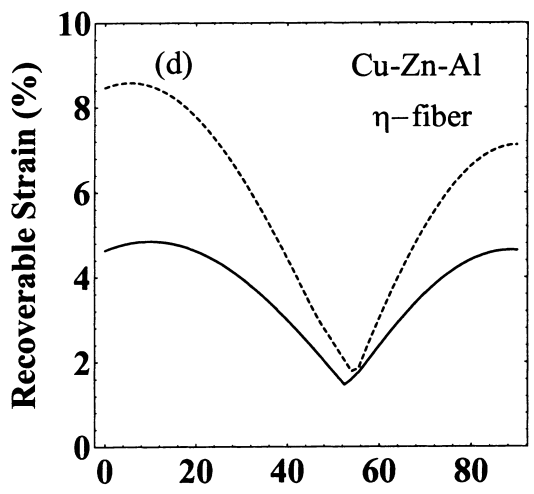

Loading Direction ( Angle from RD (deg) )

Fig. 10. The predicted uniaxial recoverable extension in $\mathrm{Cu}-\mathrm{Zn}-\mathrm{Al}$ for various rolling textures. The upper dashed line is the inner estimate $\varepsilon_{\mathrm{R}}^{\mathrm{e}}$ while the lower continuous line is the inner bound $\varepsilon_{\mathrm{R}}^{\mathrm{i}}$.

very thin films, one should use the inner bound $\varepsilon_{R}^{\text {if }}$. It turns out that they are the same for $\{110\}$ films for both $\mathrm{Ti}-\mathrm{Ni}$ and $\mathrm{Cu}-\mathrm{Zn}-\mathrm{Al}$. The prediction is consistent with the experimental observation: around $2-3.5 \%$ recoverable strain in sputter-deposited Ti-Ni thin films [37,40]. Thus, $\{110\}$ film texture is not a favorable texture in either $\mathrm{Ti}-\mathrm{Ni}$ or $\mathrm{Cu}-\mathrm{Zn}-\mathrm{Al}$.

Table 5 also lists the results for $\{100\},\{111\}$ and random films. Clearly, for the best shape-memory

Table 5. The predicted uniaxial recoverable extension for various textures. Use $\varepsilon_{\mathrm{R}}^{\mathrm{i}}$ and $\varepsilon_{\mathrm{R}}^{\mathrm{e}}$ for bulk specimens (wires, sheets, etc.) and thick films and $\varepsilon_{\mathrm{R}}^{\mathrm{if}}$ for very thin films

\begin{tabular}{|c|c|c|c|c|c|c|}
\hline \multirow[t]{3}{*}{ Texture } & \multicolumn{6}{|c|}{ Recoverable strains $(\%)$} \\
\hline & \multicolumn{3}{|c|}{$\mathrm{Ti}-\mathrm{Ni}$} & \multicolumn{3}{|c|}{$\mathrm{Cu}-\mathrm{Zn}-\mathrm{Al}$} \\
\hline & $\varepsilon_{\mathrm{R}}^{\mathrm{i}}$ & $\varepsilon_{\mathrm{R}}^{\mathrm{e}}$ & $\varepsilon_{\mathrm{R}}^{\mathrm{i} f}$ & $\varepsilon_{\mathrm{R}}^{\mathrm{i}}$ & $\varepsilon_{\mathrm{R}}^{\mathrm{e}}$ & $\varepsilon_{\mathrm{R}}^{\mathrm{i} f}$ \\
\hline random & 2.3 & 2.3 & 2.3 & 1.3 & 1.7 & 1.7 \\
\hline$\langle 110\rangle$ wire/rod & 6.0 & 7.5 & - & 1.3 & 1.7 & - \\
\hline$\langle 111\rangle$ wire/rod & 3.9 & 9.6 & - & 1.7 & 1.7 & - \\
\hline$\{111\}$ film/sheet & 4.6 & 5.3 & 8.1 & 1.4 & 1.9 & 5.9 \\
\hline$\{100\}$ film/ribbon & 2.3 & 2.3 & 2.3 & 4.6 & 7.1 & 7.1 \\
\hline$\{110\}$ sputtered film & 2.3 & 2.3 & 2.3 & 1.5 & 1.7 & 1.7 \\
\hline
\end{tabular}




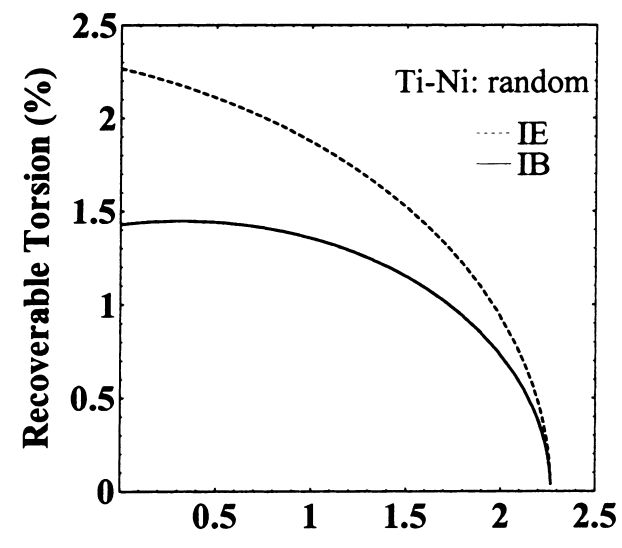

Applied Extension (\%)

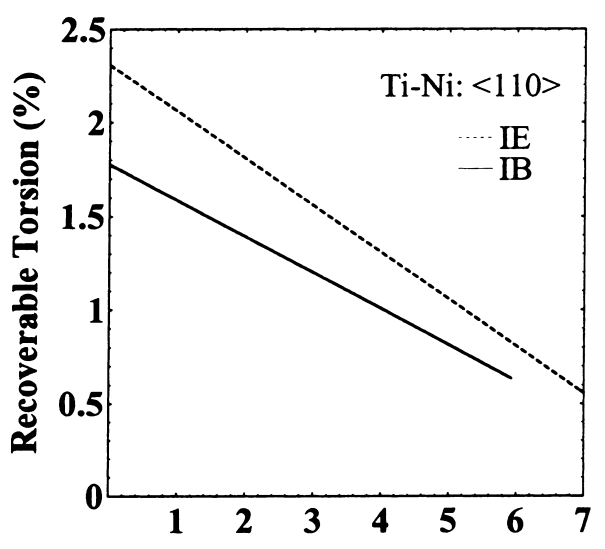

Applied Extension (\%)

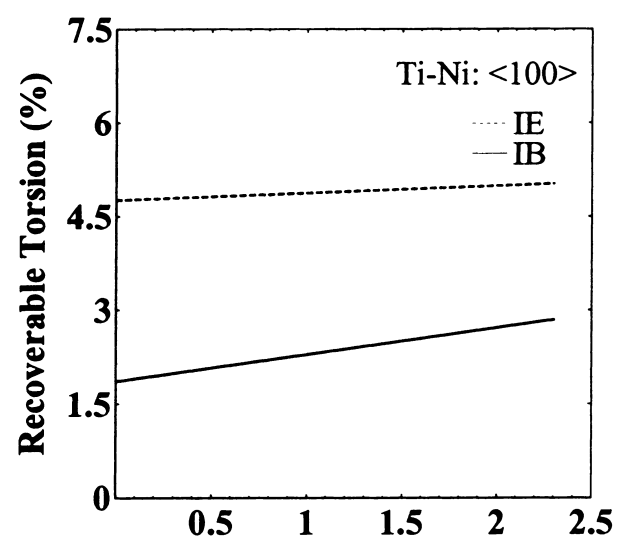

Applied Extension (\%)
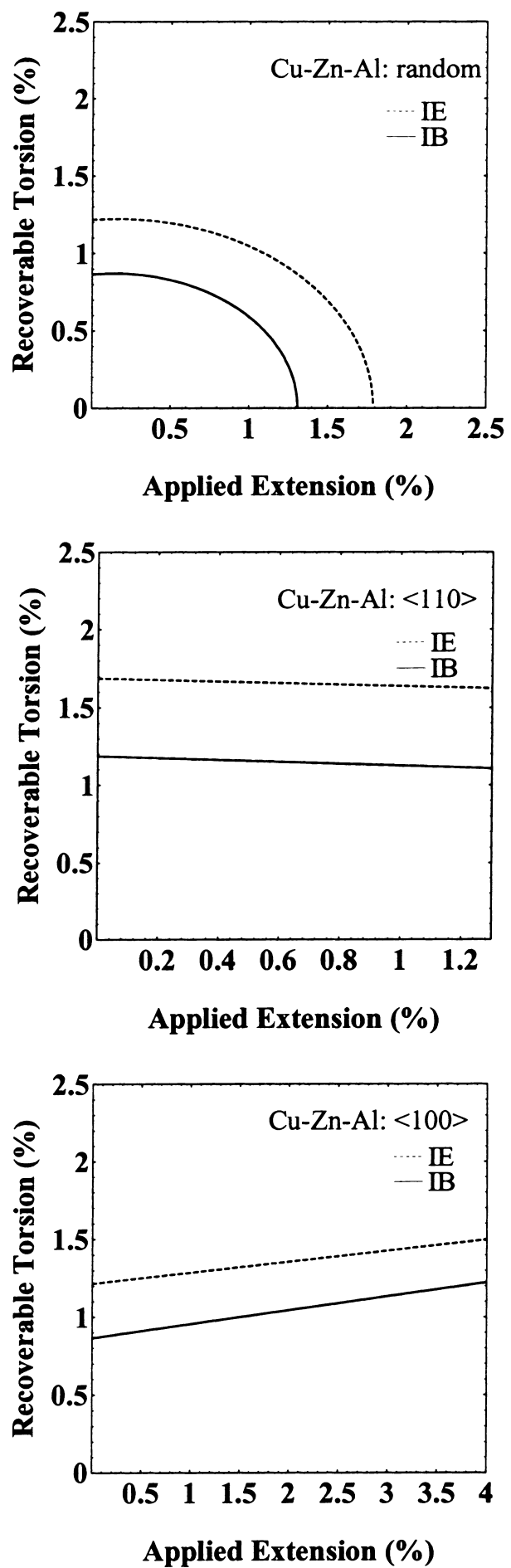

Fig. 11. The predicted recoverable torsion vs the applied extension in $\mathrm{Ti}-\mathrm{Ni}$ and $\mathrm{Cu}-\mathrm{Zn}-\mathrm{Al}$ polycrystals with random texture as well as $\langle 110\rangle$ and $\langle 100\rangle$ fiber texture. Above torsion means normalized twist or outer radius times the angle of twist. Also, IE and IB are the inner estimate $\gamma_{\mathrm{R}}^{\mathrm{e}}$ and inner bound $\gamma_{\mathrm{R}}^{\mathrm{i}}$, respectively. 
behavior one should try to make $\{111\}$ Ti-Ni films or $\{100\} \mathrm{Cu}-\mathrm{Zn}-\mathrm{Al}$ films.

\section{RESULTS FOR COMBINED TENSION-TORSION}

We now report the results for combined tensiontorsion: first pull the self-accommodated wire (or circular tube) specimen to the desired strain $\varepsilon_{0}$ below the transformation temperature, and measure the twist angle using the thermal cycling test under the constant torque. Figure 11 shows the results for a random polycrystal and for a wire or tube with $\langle 110\rangle$ or $\langle 100\rangle$ texture. Notice that the maximum recoverable normalized twists are relatively small. Further, the recoverable twist decreases with increasing imposed recoverable extension in random and $\langle 110\rangle$ texture while $\langle 100\rangle$ texture shows the opposite behavior. Sittner et al. [66] have used force control to study stress-induced pseudoelasticity in $\mathrm{Cu}-\mathrm{Zn}-\mathrm{Al}-\mathrm{Mn}$ under the combined tension-torsion. The maximum recoverable normalized twist is around $1 \%$ in their experiment, and it increases with applied tensile loading. Unfortunately they do not provide any information about the texture.

\section{DISCUSSION AND CONCLUSION}

We have investigated the effect of texture on SME. We start with a theoretical framework to quantify recoverable strain and then use inner and outer bounds to estimate it. We demonstrate with an example that the inner bound (in the case of cubic to orthorhombic transformation) accounts for the intergranular constraints and consequently provides a reasonable estimate for the actual recoverable strain while the outer bound ignores these constraints and is a large overestimate (also see Refs [32,53]). In the case of a cubic-monoclinic transformation, our inner bound becomes conservative (because we replace the unknown set $\mathscr{S}_{\text {mono }}$ with the smaller set $\mathscr{S}_{\text {orth }}$ ); so we supplement the inner bound with an inner estimate. We compare the predictions of the inner bound and estimate with the experimental observations of Zhao and Beyer [20,21] as well as Inoue et al. [18].

The inner bound and estimate are quite easy to calculate - they can be written as linear programming problems - and incorporate some easily measurable information about the texture. Therefore, these are ideal tools to study the effect of texture on SME. We demonstrate this with specific calculations focused on $\mathrm{Ti}-\mathrm{Ni}$ and $\mathrm{Cu}-\mathrm{Zn}-\mathrm{Al}$. Both these alloys undergo cubic-monoclinic transformation, recover similar strains as single crystals and are predicted to recover similar strains as random polycrystals. Yet, their observed behavior is widely varied. Figures 9 and 10 and Table 5 shed light on the striking contrast in SME between these two alloys. The following is a list of our main conclusions and suggestions.
- In $\mathrm{Ti}-\mathrm{Ni}$, the texture that develops during rolling, extrusion and drawing is extremely favorable from the point of view of large recoverable strains. In contrast, in $\mathrm{Cu}-\mathrm{Zn}-\mathrm{Al}$ and other $\mathrm{Cu}-$ based SMAs the texture that develops during these processes is rather unfavorable for SME.

- In $\mathrm{Ti}-\mathrm{Ni}$, the $\alpha$-fiber I rolling texture has the largest uniaxial recoverable strain in the RD and TD. Thus, this texture is ideal for applications which require SME in only one direction. On the other hand, the $\gamma$-fiber rolling texture has relatively large recoverable strains and has little inplane anisotropy. Hence, this texture is ideal for applications which require SME in multiple directions.

- In $\mathrm{Cu}-\mathrm{Zn}-\mathrm{Al}$ and other $\mathrm{Cu}-$ based SMAs, the SME can be improved in rolled sheets if one can make either the $\eta$-fiber texture or the $\alpha$-fiber restricted to $\{001\}\langle 110\rangle-\{112\}\langle 110\rangle$. In the later case, relatively large recoverable strain is predicted around $45^{\circ}$ from the RD.

- $\{100\}$ solidification texture shows large recoverable strain for $\mathrm{Cu}$-based SMAs but not for $\mathrm{Ti}-\mathrm{Ni}$ ribbons.

- Both Ti-Ni-based and Cu-based SMAs recover comparatively small strains in thin films owing to the unfavorable $\{110\}$ or random sputtering texture. Ti-Ni films with $\{111\}$ texture and $\mathrm{Cu}-$ based SMA films with $\{100\}$ texture are predicted to have large recoverable strains.

- Typically, the recoverable torsion is quite small. In wires, rods and tubes with random or $\langle 110\rangle$ drawing texture, the recoverable angle of twist decreases with increasing applied uniaxial extension while the behavior is reversed for $\langle 100\rangle$ texture.

We conclude with a few comments.

\subsection{Other models}

Various authors [17,19] have used a model that is equivalent to what we call the outer bound to predict the recoverable strain. This bound ignores the constraints of neighboring grains which turn out to be very important and consequently, their predictions are much larger (often by a factor of two) than the observed recoverable strains.

Inoue et al. [18] have used a different model, which they found agreed well with their observations. In the language of this paper, rather than using the set $\mathscr{S}$ for a single crystal, they use only a small subset of it, the size of which is determined by a parameter $q$. They then average over the grains; in other words they use an outer bound based on this smaller set. In the examples they consider, the underestimate in the single crystal for a choice $q=0.5$ in each grain compensates for the overestimate inherent in the outer bound, and they obtain very good agreement with experiment. However, it is unclear whether this choice gives 
good results in other situations, especially for different materials, textures and multiaxial loading.

\section{2. $R$-phase $\mathrm{Ti}-\mathrm{Ni}$}

$\mathrm{Ti}-\mathrm{Ni}$ alloys often transform from the cubic to a trigonal R-phase before transforming to the monoclinic martensite $[44,67,68]$. This cubic-trigonal transformation also displays SME and superelasticity. Though the strains are smaller, this transformation is often preferable compared to the cubicmonoclinic transformation in view of small hysteresis and ease of control [69]. According to the symmetry arguments of Bhattacharya and Kohn [1], a random polycrystal of cubic-trigonal material will not display any SME. However, a polycrystalline wire with $\langle 111\rangle$ texture has about $0.9 \%$ recoverable uniaxial strain. A rolled sheet with $\gamma$-fiber texture also has a nontrivial inner bound of about $0.2 \%$ recoverable extension in any rolling plane direction. Many shape-memory wires do possess the $\langle 111\rangle$ texture and once again this prediction is consistent with observation. Thus, texture produces the good behavior in $\mathrm{Ti}-\mathrm{Ni}$ even in the case of the R-phase.

\subsection{Texture formation}

Various researchers are conducting experiments to systematically study the effect of texture on SME. Apart from those cited above, we report on the interesting work of Matsumura et al. [70] on rolled sheets of $\mathrm{Fe}-\mathrm{Mn}-\mathrm{Si}$ alloys. This alloy undergoes a face-centered-cubic (f.c.c.) to hexagonalclose-packed (h.c.p.) transformation. By extracting specimens from different parts, Matsumura et al. have found that the surface layer with a shear texture has a larger SME than the less anisotropic mid-thickness layer. This suggests an opportunity to improve shape-memory behavior by targeting special textures using novel processing techniques.

Acknowledgements-We are grateful for helpful discussions with J. Beyer, R. V. Kohn and L. Zhao. This work was partially supported by grants from Air Force Office of Scientific Research through F49620-95-1-0109 and National Science Foundation through CMS-9457573.

\section{REFERENCES}

1. Bhattacharya, K. and Kohn, R. V., Acta mater., 1996, 44, 529.

2. Otsuka, K., Sakamoto, H. and Shimizu, K., Acta metall., 1979, 27, 585.

3. Perin, P., Bourbon, G., Goo, B. C., Charai, A., Bernardini, J. and Lexcellent, Ch., J. Physique IV, 1995, 5(C2), 263.

4. Bohong, J. and Hsu, T. Y., Mater. Sci. Forum, 1990, 56-58, 457.

5. Lexcellent, Ch. and Vacher, P., Arch. Mech., 1993, 45, 135.

6. Sakamoto, H. and Shimizu, K., Trans. Japan Inst. Metals, 1986, 27, 592.

7. Oishi, K. and Brown, L. C., Metall. Trans., 1971, 2 , 1971.

8. Eucken, S. and Hirsch, J., Mater. Sci. Forum, 1990, 56-58, 487.
9. Donner, P. and Eucken, S., Mater. Sci. Forum, 1990, 56-58, 723.

10. Miyazaki, S., Kimura, S., Otsuka, K. and Suzuki, Y., Scripta metall., 1984, 18, 883.

11. Saburi, T., Yoshida, M. and Nenno, S., Scripta metall., 1984, 18, 363.

12. Miyazaki, S., Otsuka, K. and Suzuki, Y., Scripta metall., 1981, 15, 287.

13. Piao, M., Otsuka, K., Miyazaki, S. and Horikawa, H. Mater. Trans. JIM, 1993, 34, 919.

14. Saburi, T., Tatsumi, T. and Nenno, S., J. Physique, 1982, 43(C4), 261.

15. Lin, H. C. and Wu, S. K., Acta metall., 1994, 42, 1623.

16. Li, D. Y., Wu, X. F. and Ko, T., Acta metall., 1990, 38, 19.

17. Mulder, J. H., Thoma, P. E. and Beyer, J., Z Metallk., 1993, 84, 501.

18. Inoue, H., Miwa, N. and Inakazu, N., Acta mater. 1996, 44, 4825.

19. Kitamura, K., Miyazaki, S., Iwai, H. and Kohl, M., Proc. Int. Conf. on Shape Memory and Superelastic Technologies, SMST-97, Pacific Grove, California, 1997, in press.

20. Zhao, L., Ph.D. thesis, University of Twente, The Netherlands, 1997.

21. Zhao, L. and Beyer, J., 1998, in preparation

22. Yamauchi, K., Nishida, M., Itai, I., Kitamura, K. and Chiba, A., Mater. Trans. JIM, 1996, 37, 210.

23. Willemse, P. F., Koopman, B. J. and Beyer, J., J. Physique IV, 1991, 1(C4), 329.

24. Park, N. J. and Bunge, H. J., Z. Metallk., 1990, 81, 636.

25. Park, N. J. and Bunge, H. J., J. Physique IV, 1991, $\mathbf{1}(\mathrm{C} 4), 323$.

26. Park, N. J. and Bunge, H. J., Mater. Sci. Forum, 1994, 157-162, 563.

27. Park, N. J., Wang, C. Q. and Bunge, H. J., Mater. Sci. Forum, 1994, 157-162, 827.

28. Eucken, S., Hirsch, J. and Hornbogen, E., Textures Microstruct., 1988, 8-9, 415.

29. Eucken, S., Donner, P. and Hornbogen, E., Mater. Sci. Engng, 1988, 98, 469.

30. Donner, P., J. Physique IV, 1991, 1(C4), 355.

31. Su, Q., Hua, S. Z. and Wuttig, M., J. Alloys Comp., 1994, 211/212, 460.

32. Bhattacharya, K. and Kohn, R. V., Archs Ration. Mech. Analysis, 1997, 139, 99.

33. Shu, Y. C., Ph.D. thesis, California Institute of Technology, 1998, in preparation.

34. Saburi, T. and Nenno, S., in Proc. Int. Conf. on Solid Solid Phase Transformations, ed. H. I. Aaronson, D E. Laughlin, R. F. Sekerka and C. M. Wayman. The Metall. Soc. AIME, New York, 1981, pp. 1455-1479.

35. Kohn, R. V. and Niethammer, B., 1998, in preparation.

36. Krulevitch, P., Lee, A. P., Ramsey, P. B., Trevino, J. C., Hamilton, J. and Northrup, M. A., $J$. Microelectromech. Syst., 1996, 5, 270.

37. Ishida, A., Takei, A. and Miyazaki, S., Thin Solid Films, 1993, 228, 210.

38. Miyazaki, S. and Ishida, A., Mater. Trans. JIM, 1994, $35,14$.

39. Krulevitch, P., Ramsey, P. B., Makowiecki, D. M., Lee, A. P., Northrup, M. A. and Johnson, G. C., Thin Solid Films, 1996, 274, 101.

40. Hou, L. and Grummon, D. S., Scripta metall., 1995, 33, 989.

41. Bhattacharya, K. and James, R. D., J. Mech. Phys. Solids, 1998, to be published.

42. Chakravorty, S., Ph.D. thesis, University of Illinois at Urbana-Champaign, 1975. 
43. Saburi, T. and Wayman, C. M., Acta metall., 1979, 27, 979.

44. Miyazaki, S., Kimura, S. and Otsuka, K., Phil. Mag. A, 1988, 57, 467.

45. Otsuka, K. and Shimizu, K., Trans. Japan Inst. Metals, 1974, 15, 103

46. Nam, T. H., Saburi, T., Nakata, Y. and Shimizu, K., Mater. Trans. JIM, 1990, 31, 1050

47. Knowles, K. M. and Smith, D. A., Acta metall., 1981, 29, 101.

48. Otsuka, K., Sawamura, T. and Shimizu, K., Physica status solidi (a), 1971, 5, 457.

49. Chakravorty, S. and Wayman, C. M., Acta metall., 1977, 25, 989 .

50. Otsuka, K., Nakamura, T. and Shimizu, K., Trans. Japan Inst. Metals, 1974, 15, 200.

51. Bhattacharya, K., Cont. Mech. Thermodyn., 1993, 5, 205

52. Bhattacharya, K., Archs Ration. Mech. Analysis, 1992, 120, 201.

53. Bhattacharya, K., Kohn, R. V. and Shu, Y. C., in Proc. of the IUTAM Symposium on Transformation Problems in Composite and Active Materials, ed. Y. A. Bahei-El-Din and G. J. Dvorak, 1997, in press.

54. Milton, G. W., Commun. Pure Appl. Math., 1994, 47, 959.

55. Shu, Y. C., Preprint, 1998.

56. Saburi, T., Watanabe, Y. and Nenno, S., ISIJ Int., 1989, 29, 405.
57. Bunge, H. J., Texture Analysis in Materials Science. Butterworth, London, 1982.

58. Miyazaki, S. and Otsuka, K., ISIJ Int., 1989, 29, 353.

59. Raabe, D. and Lücke, K., Mater. Sci. Forum, 1994, 157-162, 597.

60. von Schlippenbach, U., Emren, F. and Lücke, K., Acta metall., 1986, 34, 1289

61. Emren, F., von Schlippenbach, U. and Lücke, K. Acta metall., 1986, 34, 2105.

62. Mishra, S., Därmann, C. and Lücke, K., Acta metall., 1984, 32, 2185

63. Hosford, W. F., The Mechanics of Crystals and Textured Polycrystals. Oxford University Press, New York, 1993.

64. Hoogeveen, R., Moske, M., Geisler, H. and Samwer, K., Thin Solid Films, 1996, 275, 203.

65. Ying, F., Smith, R. W. and Srolovitz, D. J., Appl. Phys. Lett., 1996, 69, 3007.

66. Sittner, P., Hara, Y. and Tokuda, M., Metall. Trans., 1995, 26A, 2923.

67. Miyazaki, S. and Otsuka, K., Metall. Trans., 1986, 17A, 53.

68. Miyazaki, S. and Wayman, C. M., Acta metall., 1988 , 36, 181.

69. Otsuka, K., in Engineering Aspects of Shape Memory Alloys, ed. T. W. Duerig, K. N. Melton, D. Stöcke and C. M. Wayman. Butterworth-Heinemann, London, 1990, pp. 36-45.

70. Matsumura, O., Furusako, S., Furukawa, T. and Otsuka, H., ISIJ Int., 1996, 36, 1103. 